\title{
GABA immunostaining in the central complex of dicondylian insects
}

\author{
Uwe Homberg (iㅣ | Tim-Henning Humberg* | Jutta Seyfarth | Katharina Bode | \\ Manuel Quintero Pérez
}

Faculty of Biology, Animal Physiology \& Center for Mind, Brain and Behavior - CMBB, Philipps-Universität Marburg, Marburg, Germany

Correspondence

Uwe Homberg, Faculty of Biology, Animal Physiology, Philipps-Universität Marburg, D35032 Marburg, Germany.

Email: homberg@biologie.uni-marburg.de

Present address

*Department of Biology, University of Fribourg, $\mathrm{CH}-1700$ Fribourg, Switzerland

Funding information

Deutsche Forschungsgemeinschaft, Grant/

Award Number: HO 950/24-1

\begin{abstract}
The central complex is a group of midline-crossing neuropils in the insect brain involved in head direction coding, sky compass navigation, and spatial visual memory. To compare the neuroarchitecture and neurochemistry of the central complex in insects that differ in locomotion, ways of orientation, time of activity (diurnal, nocturnal), and evolutionary history, we studied the distribution of $\gamma$-aminobutyric acid (GABA) immunostaining in the central complex of 29 species, ranging from Zygentoma to Diptera. In all species, the lower division of the central body was densely innervated by GABA-immunoreactive tangential neurons. These neurons had additional arborizations in the bulb, a distinct region of synaptic complexes in the lateral complex, and somata in a cell cluster mediodorsally to the antennal lobe. Differences in the appearance of GABA immunostaining in the lower division of the central body corresponded to differences in neuropil architecture, such as transformation of the lower division into a toroid in certain Diptera and Heteroptera. In nearly all species two additional systems of tangential neuron of the upper division of the central body were GABA-immunoreactive. One of these systems diffusely invaded a superior layer, while the second system showed fan-like projections in an inferior layer. Sparse immunostaining in the protocerebral bridge was detected in cockroaches, a cricket, and two hemipteran species. The data show that three systems of GABA-immunoreactive tangential neurons of the central body are highly conserved and suggest that the layered organization of the upper division of the central body is, likewise, largely maintained from basal to advanced species.
\end{abstract}

\section{KEYWORDS}

immunocytochemistry, insect brain, neuroanatomy, pancrustacean phylogeny, RRID:

AB_2314457, RRID: AB_2315056, RRID: AB_261363, spatial orientation, $\gamma$-aminobutyric acid

\section{1 | INTRODUCTION}

The central complex is a group of midline spanning neuropils in the center of the insect brain (Homberg, 2008; Pfeiffer \& Homberg, 2014; Strausfeld, 1976). Accumulating evidence from anatomical studies, intracellular and extracellular recordings, lesion experiments, and, in Drosophila, behavioral studies of mutant and transgenic animals points to a role of the central complex in various aspects of spatial orientation. Central-complex lesions in cockroaches affected turning behavior and obstacle avoidance while electrical stimulations led to changes in locomotor activity
(Ritzmann et al., 2012). Behavioral analysis of mutant and transgenic flies indicate a key role in the directional control of walking and climbing (Poeck, Triphan, Neuser, \& Strauss, 2008; Triphan, Poeck, Neuser, \& Strauss, 2010), spatial orientation memory (Neuser, Triphan, Mronz, Poeck, \& Strauss, 2008), visual pattern memory (Liu et al., 2006; Wang et al., 2008), and place learning (Ofstad, Zuker, \& Reiser, 2011). Visual signals seem to be the dominant sensory input in many species. In locusts, crickets, monarch butterflies, dung beetles, and bees, central-complex neurons code for the plane of polarized light from zenithal directions suggesting a role in sun compass orientation based on the polarization pattern of the sky (el Jundi 
et al., 2015; Heinze \& Reppert, 2011; Sakura, Lambrinos, \& Labhart, 2008; Stone et al., 2017; Vitzthum, Müller, \& Homberg, 2002). Extracellular recordings in cockroaches and $\mathrm{Ca}$-imaging experiments in flies indicate that certain central-complex neurons signal heading direction based on visual panorama information and self-generated proprioceptive input (Seelig \& Jayaraman, 2013, 2015; Varga \& Ritzmann, 2016). Somewhat unrelated to this is an established role in sleep homeostasis in flies (Donlea et al., 2018; Liu, Liu, Tabucchi, \& $\mathrm{Wu}, 2016$ ). In all pterygote insects studied so far, four subunits of the central complex have been recognized; the protocerebral bridge $(\mathrm{PB})$, the upper and lower divisions of the central body (CBU, CBL; also termed fan-shaped body and ellipsoid body, respectively) and a pair of ventral noduli (Pfeiffer \& Homberg, 2014). The central complex is closely associated with ventrolaterally adjacent neuropils of the brain, termed the lateral complex. Internally, the PB and the central body $(\mathrm{CB})$ are subdivided into linear arrays of 16-18 slices and the subdivisions of the $\mathrm{CB}$, in addition, into horizontal layers. Three categories of neuronal cell types have been described in flies (Hanesch, Fischbach, \& Heisenberg, 1989; Wolff, lyer, \& Rubin, 2015; Young \& Armstrong, 2010), locusts (Heinze \& Homberg, 2008; Müller, Homberg, \& Kühn, 1997), monarch butterflies (Heinze, Florman, Asdokaraj, el Jundi, \& Reppert, 2013), and, less completely, in several other species: tangential neurons, columnar neurons, and pontine neurons. Tangential neurons have arborizations in a variety of brain areas outside the central complex and ramify throughout the $\mathrm{PB}$, in particular layers of the $\mathrm{CB}$, or in the noduli. Most of these neurons are regarded as input elements to the central-complex network. Pontine neurons interconnect single slices usually across the midline and have only been found in the CBU. Several dozen systems of columnar neurons, each consisting of 16 elements, finally, connect slices of the central-complex subunits in a topographic way and form regular fiber crossings between the right and left hemispheres of the central complex. These neurons provide output from the central complex to the lateral accessory lobes and other areas of the brain.

Corresponding to the large variety of neuronal cell types, many neurotransmitters and neuropeptides have been demonstrated through immunostaining or histochemical staining in the central complex. These include $\gamma$-aminobutyric acid (GABA), the biogenic amines dopamine, serotonin, histamine, and octopamine, nitric oxide, and neuropeptides related to more than a dozen peptide families (Pfeiffer \& Homberg, 2014). GABA is a major inhibitory transmitter in the insect brain (Buckingham, Biggin, Sattelle, Brown, \& Sattelle, 2005; Lummis, 1990). In all species studied, including grasshoppers (Homberg, Vitzthum, Müller, \& Binkle, 1999; Kunst, Pförtner, Aschenbrenner, \& Heinrich, 2011), the house cricket (Strambi et al., 1998), the tobacco hawk moth (Homberg, Kingan, \& Hildebrand, 1987), the American cockroach (Blechschmidt, Eckert, \& Penzlin, 1990), the honeybee (Mota et al., 2016; Schäfer \& Bicker, 1986), and two species of flies (Hanesch et al., 1989; Meyer, Matute, Streit, \& Nässel, 1986), the CBL showed dense GABA immunostaining. Detailed analysis in the locust Schistocerca gregaria, the fly Drosophila melanogaster, and the moth Manduca sexta identified tangential neurons of the CBL (termed TL neurons in locusts, $R$ neurons in flies) to be immunostained. These neurons have dendritic arborizations in microglomerular complexes in the bulbs, small areas in the lateral complex (Held et al., 2016; Schmitt, Stieb, Wehner, \& Rössler, 2016; Träger, Wagner, Bausenwein, \& Homberg, 2008). In addition, in all species studied, the CBU showed sparse GABA immunoreactivity. In the most detailed study on the desert locust, GABA immunostaining in the $C B U$ was attributed to two types of tangential neuron with presumed dendritic ramifications in the lateral accessory lobes (Homberg et al., 1999). One of these cell types gave rise to prominently beaded ramifications in the most superior layer of the $\mathrm{CBU}$, while the second type had finer terminals in an intermediate layer.

To obtain further data on the evolution and functional roles of the central complex across insects, we analyzed GABA immunostaining in representatives from 12 insect orders with particular focus on species that differ in ways of locomotion, time of activity (diurnal and nocturnal), and ways of orientation. The data show that the pattern of GABA immunostaining in the central complex is remarkably conserved throughout dicondylian insects. Changes from a common ground pattern corresponding to changes in neuropil architecture have occurred in Diptera and certain Heteroptera, differences in staining pattern, in certain Orthoptera and Blattaria.

\section{2 | MATERIAL AND METHODS}

\section{1 | Insect species}

Experiments were performed on 29 species of dicondylian insects (Table 1). Firebrats (Thermobia domestica), cockroaches (Rhyparobia maderae, Blaberus discoidalis, Periplaneta americana), honeybees (Apis mellifera), meal worm beetles (Tenebrio molitor), sphinx moths (Manduca sexta), and yellow fever mosquitoes (Aedes aegypti) were obtained from laboratory cultures at the Department of Biology. Firebrats were raised in an incubator $\left(37^{\circ} \mathrm{C}\right)$ in constant darkness. Cockroaches were reared under $12 \mathrm{~L}: 12 \mathrm{D}$ photoperiod and a temperature of $27^{\circ} \mathrm{C}$. Mosquitoes were reared under $12 \mathrm{~L}: 12 \mathrm{D}$ photoperiod, at about $70 \%$ humidity and a temperature of $27^{\circ} \mathrm{C}$, and sphinx moth were reared as described previously (Homberg et al., 1987). Praying mantises (Hierodula membranacea), blowflies (Lucilia sericata), house crickets (Acheta domesticus), assassin bugs (Psytalla horrida), sun beetles (Pachnoda marginata), and butterflies (Danaus plexippus, Eryphanis polyxena) were obtained from commercial suppliers. The flies were obtained as larvae and were raised at $30{ }^{\circ} \mathrm{C}$ in constant darkness, house crickets and assassin bugs were kept at room temperature. Backswimmers (Notonecta glauca), water striders (Gerris lacustris), water beetles (Acilius sulcatus), and dragonfly larvae (Aeshna cyanea, Sympetrum vulgatum) were caught in local ponds. Some larval A. cyanea were allowed to eclose to adulthood from a laboratory aquarium. Earwigs (Forficula auricularia), wasps (Vespula vulgaris, Paravespula germanica), green shield bugs (Palomena prasina), june beetles (Amphimallon solstitiale), ants (Formica rufa), scorpionflies (Panorpa communis), craneflies (Tipula oleracea), and flesh flies (Sarcophaga carnaria) were caught outdoors near the Department of Biology, University of Marburg. Dragonflies and water beetles were studied as larvae and adults, all other insects were studied exclusively as adults. All animal procedures were in compliance with the guidelines of the 
TABLE 1 List of species, including the order and family they belong to, for which GABA immunostaining in the central complex has been characterized here and in previous studies

\begin{tabular}{|c|c|c|}
\hline Order & Family & Species \\
\hline Zygentoma & Lepismatidae & Thermobia domestica \\
\hline \multirow[t]{2}{*}{ Odonata } & Aeshnidae & Aeshna cyanea \\
\hline & Libellulidae & Sympetrum vulgatum \\
\hline Dermaptera & Forficulidae & Forficula auricularia \\
\hline \multirow[t]{3}{*}{ Orthoptera } & Acrididae & $\begin{array}{l}\text { Schistocerca gregaria }{ }^{a} \text { (Homberg } \\
\text { et al., 1999) }\end{array}$ \\
\hline & Acrididae & $\begin{array}{l}\text { Chorthippus biguttulus }{ }^{a} \text { (Kunst et al., } \\
\text { 2011) }\end{array}$ \\
\hline & Ensifera & $\begin{array}{l}\text { Acheta domesticus (Strambi et al., } \\
\text { 1998) }\end{array}$ \\
\hline Mantodea & Mantidae & Hierodula membranacea \\
\hline \multirow[t]{3}{*}{ Blattodea } & Blattidae & $\begin{array}{l}\text { Periplaneta americana (Blechschmidt } \\
\text { et al., 1990) }\end{array}$ \\
\hline & Blaberidae & Rhyparobia maderae \\
\hline & Blaberidae & Blaberus discoidalis \\
\hline \multirow[t]{4}{*}{ Hemiptera } & Pentatomidae & Palomena prasina \\
\hline & Reduviidae & Psytalla horrida \\
\hline & Notonectidae & Notonecta glauca \\
\hline & Gerridae & Gerris lacustris \\
\hline \multirow[t]{4}{*}{ Hymenoptera } & Apidae & $\begin{array}{l}\text { Apis mellifera (Schäfer \& Bicker, } \\
\text { 1986) }\end{array}$ \\
\hline & Vespidae & Vespula vulgaris \\
\hline & Vespidae & Paravespula germanica \\
\hline & Formicidae & Formica rufa \\
\hline \multirow[t]{4}{*}{ Coleoptera } & Tenebrionidae & Tenebrio molitor (Wegerhoff, 1999) \\
\hline & Dytiscidae & Acilius sulcatus \\
\hline & Scarabaeidae & Pachnoda marginata \\
\hline & Scarabaeidae & Amphimallon solstitiale \\
\hline \multirow[t]{3}{*}{ Lepidoptera } & Sphingidae & $\begin{array}{l}\text { Manduca sexta (Homberg et al., } \\
\text { 1987) }\end{array}$ \\
\hline & Nymphalidae & Danaus plexippus \\
\hline & Nymphalidae & Eryphanis polyxena \\
\hline Mecoptera & Panorpidae & Panorpa communis \\
\hline \multirow[t]{6}{*}{ Diptera } & Culicidae & Aedes aegypti \\
\hline & Tipulidae & Tipula oleracea \\
\hline & Drosophilidae & $\begin{array}{l}\text { Drosophila melanogaster }{ }^{a} \text { (Hanesch } \\
\text { et al., 1989) }\end{array}$ \\
\hline & Muscidae & $\begin{array}{l}\text { Musca domestica }{ }^{a} \text { (Meyer et al., } \\
\text { 1986) }\end{array}$ \\
\hline & Calliphoridae & Lucilia sericata \\
\hline & Sarcophagidae & Sarcophaga carnaria \\
\hline
\end{tabular}

a Species not examined in this study.

European Union (Directive 2010/63/EU) and the German Animal Welfare Act.

\section{2 | Immunocytochemistry of GABA}

Immunocytochemical staining for GABA was performed on vibratome or paraffin sections using the indirect peroxidase or the peroxidaseantiperoxidase (PAP) technique (Sternberger, 1979). Animals were anesthetized by cooling to $4{ }^{\circ} \mathrm{C}$ or on ice. Brains were dissected and fixed in a solution containing one part (by volume) $25 \%$ glutaraldehyde and three parts saturated picric acid and adding acetic acid to a final concentration of 1\% (GPA-fixative; Boer et al., 1979). Fixation lasted for about $4 \mathrm{hr}$ at room temperature or overnight at $4{ }^{\circ} \mathrm{C}$. For staining on paraffin sections, brains were dehydrated through an ascending series of ethanol solutions, transferred to toluene, and embedded in paraffin (Paraplast Plus; Sigma, Steinheim, Germany). Brains were sectioned with a rotary microtome (Leitz, Wetzlar, Germany) at $8 \mu \mathrm{m}$ or $10 \mu \mathrm{m}$. Sections were dried onto albumin-glycerin coated microscope slides on a hot plate at about $40^{\circ} \mathrm{C}$. Sections were deparaffinized through xylene, hydrated through a series of ethanol solutions and were, finally, incubated in $0.1 \mathrm{M}$ Tris $\mathrm{HCl} / 0.3 \mathrm{M} \mathrm{NaCl}$ (SST) containing $0.1 \%$ Triton $\mathrm{X}-100$ ( $\mathrm{TrX}), \mathrm{pH}$ 7.4. For staining on vibratome sections, the fixed brains were embedded in gelatin-albumin as described by Homberg et al. (1999). Brains were sectioned at $30 \mu \mathrm{m}$ with a vibrating-blade microtome (VT1200S, Leica, Wetzlar, Germany).

Sections were treated for GABA immunreactivity as described by Hoskins, Homberg, Kingan, and Hildebrand (1987) and Homberg et al. (1999). To reduce nonspecific staining, brain sections were preincubated for $1 \mathrm{hr}$ in SST with 0.5\% TrX-100 containing 5\% normal goat serum (NGS). Incubation in GABA antiserum, diluted at 1:1,000 on paraffin sections and 1:6,000-1:10,000 on vibratome sections in SST, 0.5\% TrX and 5\% NGS was carried out for $20 \mathrm{hr}$ at room temperature. Following incubation in primary antibody, the preparations were washed in SST with $0.1 \% \operatorname{TrX}$ and were incubated for $1 \mathrm{hr}$ at room temperature in secondary antibody, goat anti-rabbit IgG (RRID: AB_261363) diluted 1:40 in SST, 0.5\% TrX and 1\% NGS. After rinsing in SST, 0.1\% TrX, the sections were treated for $1 \mathrm{hr}$ at room temperature in rabbit PAP (RRID: AB_2315056) at 1:300 in SST 0.5\% TrX-100 containing $1 \%$ NGS. Sections were stained by incubation in a solution of $3,3^{\prime}$-diaminobenzidine tetrahydrochloride (DAB, $0.03 \mathrm{mg} / \mathrm{ml}$ ) in $0.1 \mathrm{M}$ phosphate buffer $(\mathrm{pH} 7.4)$, and $\mathrm{H}_{2} \mathrm{O}_{2}(0.02 \%)$. In some experiments, staining was intensified by adding nickel ammonium sulfate ( $3 \%$ in water) to the staining solution $(0.03 \mathrm{mg} / \mathrm{ml}$ DAB in $0.5 \mathrm{M}$ Tris$\mathrm{HCl}, \mathrm{pH} 7.4$ and $0.02 \% \mathrm{H}_{2} \mathrm{O}_{2}$ ) resulting in a dark blue reaction product. After staining, the sections were washed, dehydrated, cleared, and mounted in Entellan (Merck, Darmstadt, Germany) under glass cover slips.

In backswimmers and wasps, the indirect peroxidase technique was used. Following incubation with the GABA antiserum, the sections were incubated for $1 \mathrm{hr}$ with peroxidase conjugated goat anti-rabbit (Dianova, Hamburg, Germany) at 1:300 in SST 0.1\% TrX, containing $5 \%$ NGS. The DAB reaction was carried out as described above.

\section{3 | Antibody characterization}

The GABA antiserum (\# 9/24, provided by Dr. T. G. Kingan, RRID: $\left.A B \_2314457\right)$ is an affinity-purified antiserum raised in rabbit against conjugates of GABA coupled via glutaraldehyde to keyhole limpet hemocyanin $(\mathrm{KLH})$. Immunostaining on brain sections of the sphinx moth $M$. sexta was abolished after preadsorption of the antiserum with $24 \mathrm{nM}$ GABA-KLH conjugates but no reduction in staining occurred following preadsorption with conjugates of L-glutamic acid, $\beta$-alanine, L-glutamine, and taurine (Hoskins, Homberg, Kingan, \& 


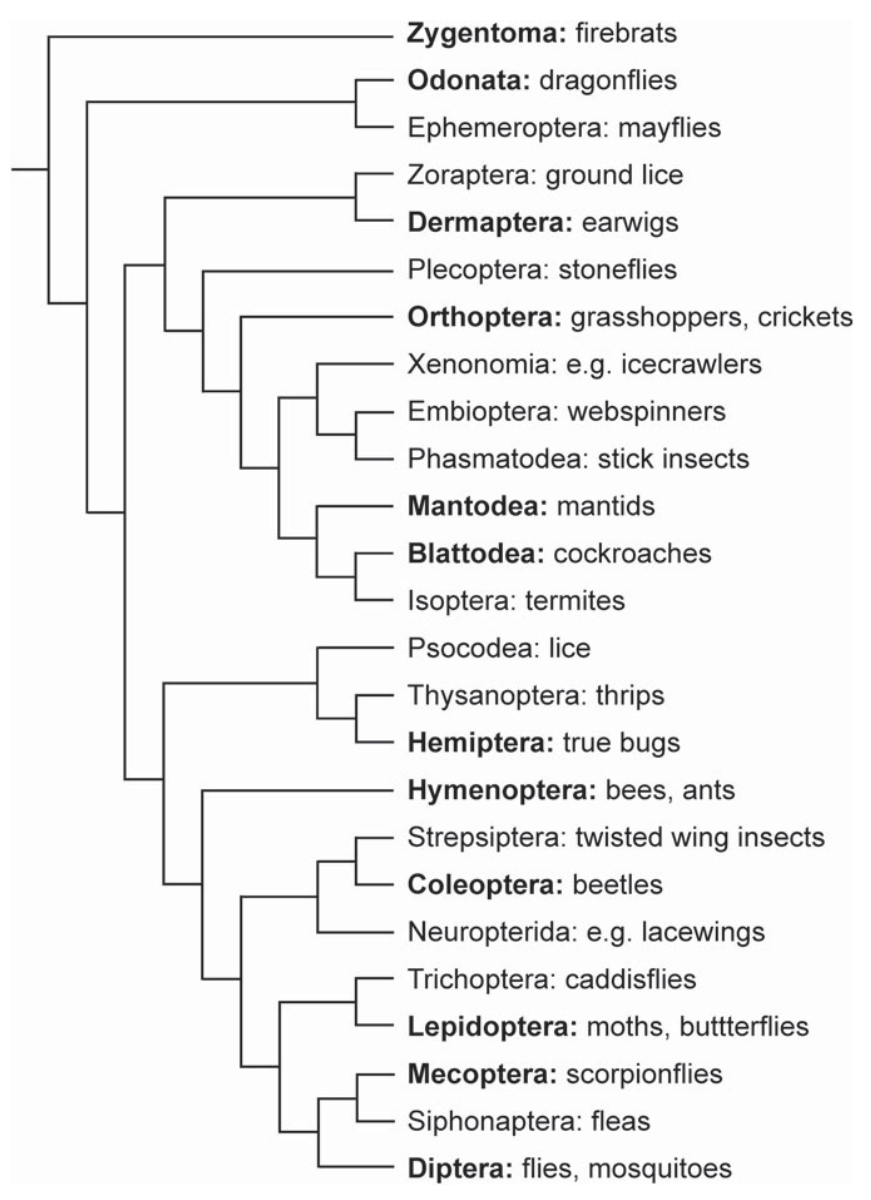

FIGURE 1 Phylogenetic tree illustrating the relationship between insect orders based on data from Trautwein, Wiegmann, Beutel, Kjaer, and Yeates (2012) and Misof et al. (2014). Representatives from orders in bold were examined here and/or in previous studies

Hildebrand, 1986). Preadsorption of the diluted antiserum with 15 nM GABA-BSA conjugate abolished all immunostaining on vibratome sections of the brain of the desert locust $S$. gregaria (Homberg et al., 1999), and with $50 \mu \mathrm{M}$ GABA-glutaraldehyde complex on brain sections of the praying mantis $\mathrm{H}$. membranacea (Rosner, von Hadeln, Salden, \& Homberg, 2017). Finally, a second antiserum against GABA (\#4TB, produced by Dr. H. Dircksen) and an antiserum against the GABA-synthesizing enzyme glutamate decarboxylase raised in sheep (generated by Dr. W. H. Oertel, Munich) resulted in immunostaining of locust brain sections indistinguishable in their pattern of labeling from that obtained with the 9/24 antiserum used here (Homberg et al., 1999).

\section{4 | Image acquisition and reconstructions}

Images from selected sections were captured by a digital camera (ProgRes C12plus, Jenoptik) mounted on a transmission light microscope (Axioskop, Zeiss, Oberkochen, Germany). Images were optimized in contrast and brightness using Photoshop CS3 and CC 2017 (Adobe Systems, San Jose, CA). Some figures show superimposed images from two to three adjacent sections (see Figure legends). In Figures $8 \mathrm{j}$ and $10 \mathrm{a}$ images were obtained by montaging adjacent areas from the sections using the "darken" function of Adobe Photoshop.
Immunostained neurons of the CBL and CBU were traced from serial brain sections through a camera lucida attachment on a compound microscope (Leitz, Wetzlar, Germany). The drawings were digitized with a scanner (CanoScan 9000F Mark II, Canon, Tokyo, Japan). Images and the 2D-reconstructions were assembled in CorelDraw software (Ottawa, Ontario, Canada). Positional information in all figures is given with regard to the animal's body axis.

\section{3 | RESULTS}

Table 1 lists all species that were examined for GABA immunoreactivity in the central complex in this and previous studies. To detect possible evolutionary changes in GABA-immunoreactive fiber systems, a broad range of dicondylian species were selected covering basal and advanced orders. Figure 1 illustrates the phylogeny of insect orders based on Trautwein et al. (2012) and Misof et al. (2014), which indicates the relationships of species studied here and in previous accounts. Given the prominent role of this brain area in spatial orientation, species were furthermore selected for differences in their mode of locomotion (walking, flying, swimming), phase of activity (nocturnal, diurnal), and visual performance (eye size and usage), in order to detect possible adaptations of GABA-immunoreactive fiber systems to different lifestyles.

\section{1 | GABA immunostaining in the central complex of the firebrat (Zygentoma)}

A single species, the firebrat Thermobia domestica, was studied of the most basal order of dycondylian insects, the Zygentoma. The CB of $T$. domestica had a crescent shape. GABA immunostaining revealed a bisection into a densely immunostained lower division (CBL) and a more sparsely stained upper division (CBU) of the $C B$ (Figure 2). Dense GABA immunostaining in the $\mathrm{CBL}$ originated from small groups of cell bodies between the antennal lobe and the lateral accessory lobe. Neurons formed a small fiber fascicle, the isthmus tract that passed posteriorly around the lobes of the mushroom body and entered the CBL along its ventral face. Small side branches were present near the lobes of the mushroom body. Diffuse, beaded GABA immunoreactivity was present throughout the $\mathrm{CBU}$, but its cellular origin could not be resolved (Figure 2). Staining in a layer adjacent to the $\mathrm{CBL}$ was slightly denser than in the rest of the CBU. No immunostaining was detected in the PB. Noduli could not be identified.

\section{2 | GABA immunostaining in the central complex of dragonflies (Odonata)}

Two species of dragonfly were studied, the southern hawker, Aeshna cyanea and the vagrant darter Sympetrum vulgatum, both in their larval stage, and A. cyanea, in addition, as freshly eclosed adults. GABA immunostaining in both species had a similar pattern although the shape of the CB differed slightly (Figure 3). Likewise, the pattern of immunostaining in larval and adult $A$. cyanea was highly similar (Figure $3 a-g$ ). In both species, the CBL was densely immunostained. In addition, two layers of the CBU, a dorsalmost layer (GU1) and a 

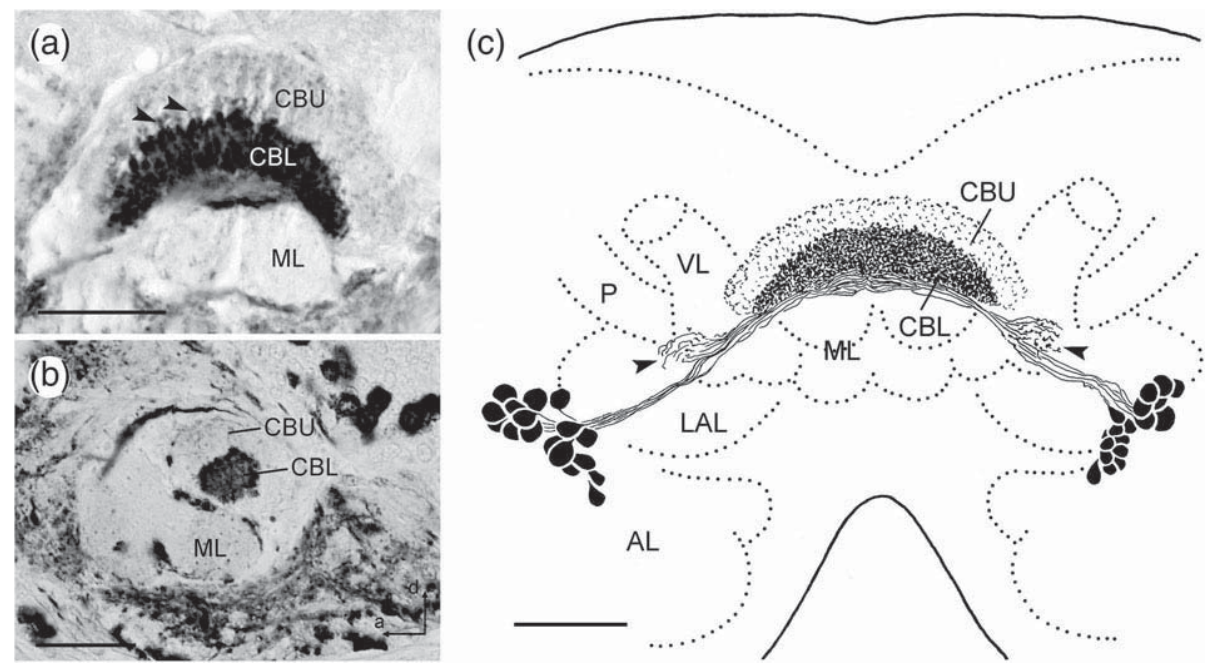

FIGURE $2 \gamma$-Aminobutyric acid (GABA) immunostaining in the central complex of the firebrat Thermobia domestica. (a) Frontal paraffin section through the central body. (b) Mid-sagittal section through the central body. The lower division of the central body (CBL) shows dense GABA immunostaining, the upper division (CBU) is only sparsely stained. A narrow layer of the $C B U$ above the $C B L$ shows slightly stronger immunostaining than the rest of the CBU (arrowheads in a). (c) Frontal reconstruction of GABA-immunoreactive neurons associated with the central body. Neurons have cell bodies in a cluster between the antennal lobe and the lateral accessory lobe. Short side branches innervate small areas (arrowheads) posterior to the origin of the medial lobe of the mushroom body (ML). a, anterior; $d$, dorsal; LAL, lateral accessory lobe; $\mathrm{P}$, pedunculus; VL, vertical lobe of the mushroom body. Scale bars $=50 \mu \mathrm{m}$

ventral layer (GU2) showed less dense immunolabeling. The noduli and PB were unlabeled. Staining in the $\mathrm{CBL}$ and the two $\mathrm{CBU}$ layers originated from tangential neurons entering the $\mathrm{CB}$ through the isthmus tracts (Figure 3b,d,e,f,h,i). Immunostained cell bodies were clustered anterolaterally from the lateral accessory lobes (Figure 3d). Neurons innervating the CBL had dense knot-like terminals in the bulbs lateral from the CB (Figure $3 c, d$ ). Fibers continued as a loose bundle toward the brain midline and gave rise to dense immunostaining of palisade-like appearance throughout the CBL (Figure 3b,d,e,g,h). $A$ few fibers running anterior from the $C B L$ gave rise to processes ascending along the anterior face of the CBL and innervated an uppermost layer of the $\mathrm{CBU}$ with diffusely distributed beaded endings (Figure 3b,d). These neurons were termed type 1 GABAimmunoreactive neurons of the CBU (GU1). In S. vulgatum, these neurons sent major processes to the upper margin of the $\mathrm{CBU}$ from where fine neurites descended into the superior layer in an umbrellalike manner (Figure 3i). Neurons innervating the lower layer of the $\mathrm{CBU}$, termed GU2, instead, projected from the common fiber bundle below the $C B L$ in a fan-like manner posterior from the $C B L$ to the $\mathrm{CBU}$ and gave rise to a fan-shaped appearance of staining in the ventral layer (Figure 3f,i).

\subsection{GABA immunostaining in the central complex of an earwig (Dermaptera) and the house cricket (Orthoptera)}

The CB of the earwig Forficula auricularia had a hemiellipsoidal shape (Figure 4a,b). Fibers from GABA-immunoreactive neurons near the antennal lobe extended through the isthmus tract, innervated the $C B$ ventrolaterally, and gave rise to dense staining throughout the $C B L$, concentrated in patch-like condensations (Figure 4b). Ramifications in the lateral complex were inconspicuous but concentrated in a small area corresponding to the bulb laterally near the antennal lobe. Side branches from the midline-crossing bundle ventrally from the $C B$ extended dorsally along the anterior surface of the $C B$ and gave rise to beaded terminals throughout the CBU (Figure 4a,b). Based on their similar path of entry into the $\mathrm{CBU}$, these neurons were termed, as in dragonflies, GU1 neurons. A more sharply defined layer of the CBU, near the dorsal face of the CBL, was more densely labeled, but the origin of its processes could not be revealed (Figure 4a). These neurons were labeled GU2 neurons. No immunostaining was detected in the noduli or the $\mathrm{PB}$.

GABA immunostaining in the cricket Acheta domesticus was described by Strambi et al. (2015) but here we reveal additional details. As in all other species studied, the CBL showed strongest immunolabeling. Staining resulted from cell bodies distributed in the soma rind adjacent to the antennal lobe (Figure 4d). Neurons gave rise to two clusters of microglomerular condensations in the lateral complex, one cluster more laterally termed lateral bulb and the second, adjacent to the CB, termed medial bulb (Figure 4d,f). From the common isthmus tract below the $\mathrm{CB}$, a fascicle separated and crossed the brain midline along the anterior dorsal face of the CBL (arrowheads in Figure 4e,f). From this bundle, fine fiber fascicles projected dorsally into the CBU separating this part of the CBU into eight segments (Figure $4 \mathrm{~d}, \mathrm{f}, \mathrm{g}$ ), and gave rise to fine beaded processes diffusely distributed throughout the dorsofrontal part of the CBU (labeled GU1 in Figure 4e). A second system of neurons originating from the fiber complex ventrally of the $C B L$ extended processes posteriorly from the $C B L$ into a posterior-ventral layer of the CBU (labeled GU2 in Figure 4e,g). Their terminals had a varicose appearance. In addition, the PB was tangentially invaded by 2-3 fibers from its lateral tips that gave rise to sparse beaded processes 

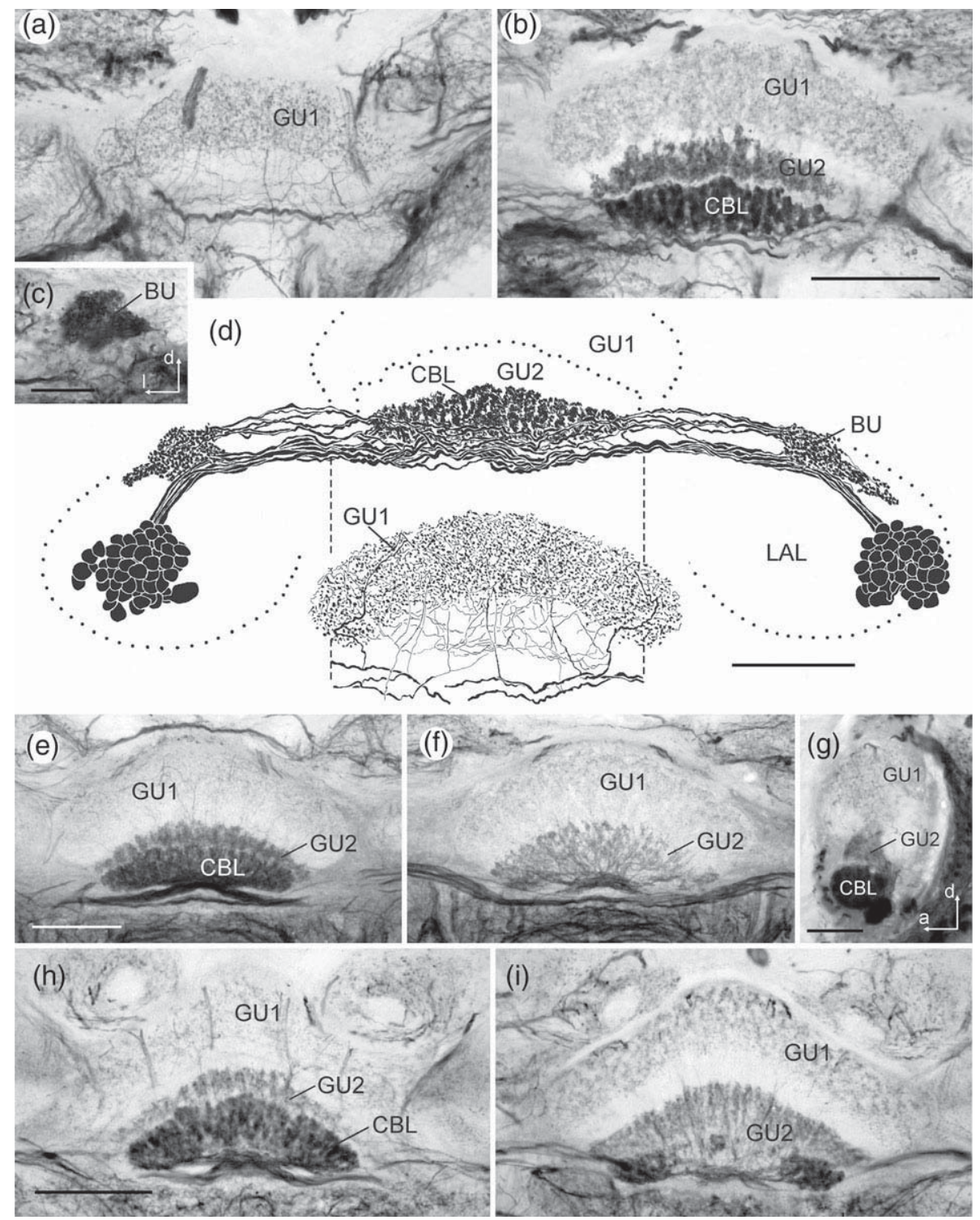

FIGURE 3 GABA immunostaining in the central complex of dragonflies. (a-d) Aeshna cyanea, larva, (e-g) Aeshna cyanea, adult, (h, i) Sympetrum vulgatum, larva. ( $a, b)$ Frontal sections through the central body at an anterior (a) and a more posterior level (b). The lower division (CBL) and two layers of the upper division of the central body (GU1, GU2) show GABA immunostaining. Dense GABA immunoreactivity in the CBL has a palisade-like appearance. (c) The bulb of the lateral complex (BU) shows dense immunolabeling. (d) Frontal reconstruction of GABA-immunoreactive neurons innervating the CBL and GU1 neurons of the CBU. The immunostained neurons have cell bodies in clusters anterior-ventrally to the lateral accessory lobes. $(\mathrm{e}-\mathrm{g})$ Frontal sections $(\mathrm{e}, \mathrm{f})$ and sagittal section $(\mathrm{g})$ through the central body of adult $A$. cyanea. The pattern of immunostaining is highly similar to that in the larva. The GU2 processes enter the upper division of the central body ( $\mathrm{f}$ ) in a fan-shaped manner. (h, i) $\mathrm{S}$. vulgatum larva at an anterior (h) and more posterior level (i). Like in A. cyanea, the CBL and two layers of the CBU (GU1, GU2) show GABA immunostaining. a, anterior; d, dorsal; I, lateral. Scale bars $=100 \mu \mathrm{m}$ in b (also applies to a), d, e (also applies to f), h (also applies to i); $50 \mu \mathrm{m}$ in c, g

throughout the bridge (Figure 4c,e). The noduli were free of immunostaining (Figure 4g)

\subsection{GABA immunostaining in the central complex of a praying mantis (Mantodea) and cockroaches (Blattodea)}

We studied immunostaining in the praying mantis Hierodula membranacea and three species of cockroach, the Madeira cockroach Rhyparobia maderae and the discoid cockroach Blaberus discoidalis (both Blaberidae) and reinvestigated immunostaining in the American cockroach Periplaneta americana (Blattidae) (Table 1). In all species, three apparently homologous systems of neuron exhibited immunostaining, tangential neurons of the $\mathrm{CBL}$ and two types of tangential neuron of the CBU (Figure 5). In the praying mantis, the $C B L$ showed dense immunostaining with a palisadelike pattern (Figure $5 \mathrm{a}$ ). In addition, a few fibers projecting horizontally along the dorsofrontal surface of the CBU gave rise to diffuse immunostaining in a superficial layer of the CBU (Figure 5a,b; GU1). Finally, a layer adjacent to the dorsal surface of the CBL was invaded by immunostained processes in a fan-shaped manner (GU2) with staining concentrated in 10 distinct domains (labeled 1-5 in Figure 5a,b). All processes could be traced to fibers in the isthmus tracts and cell bodies dorsomedial from 

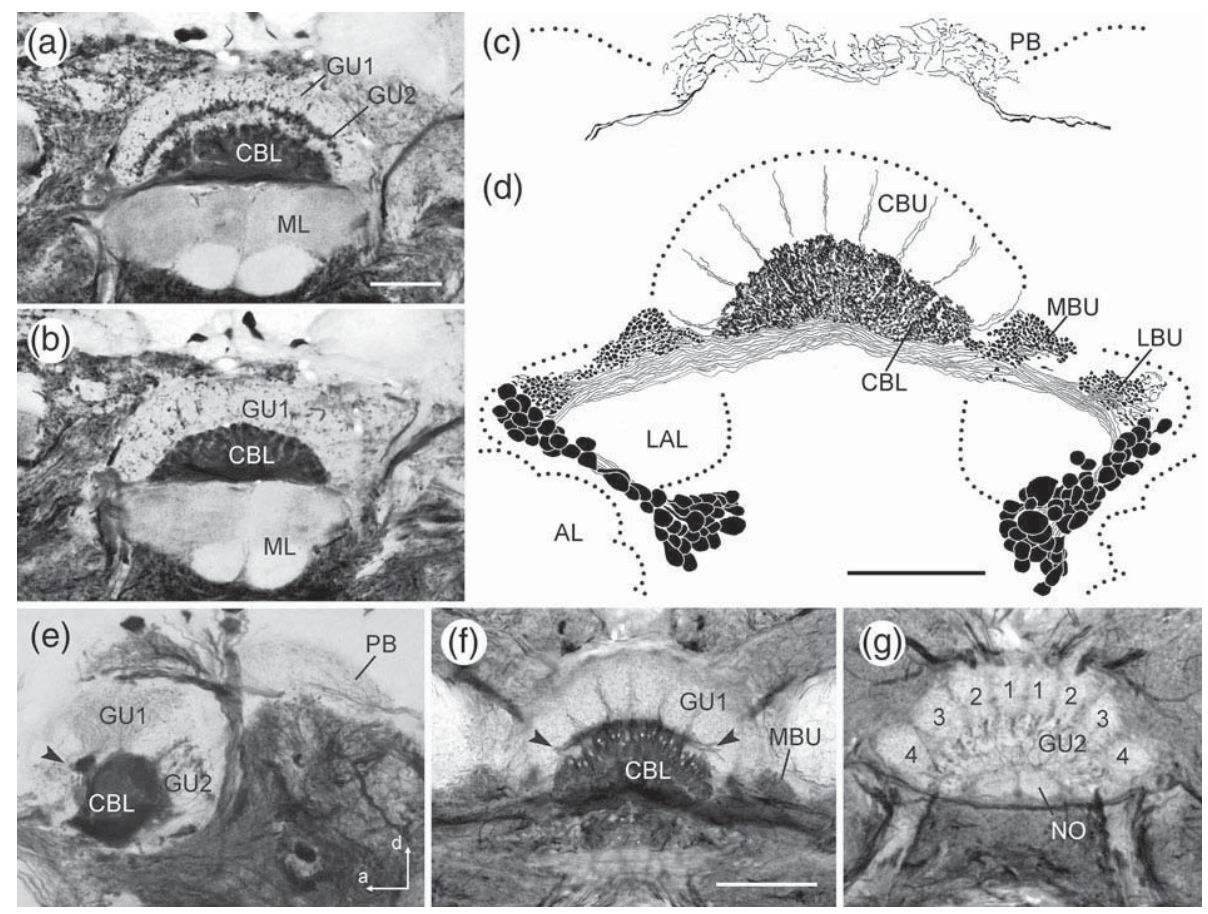

FIGURE 4 GABA immunostaining in the central complex of Dermaptera and Orthoptera. (a, b) Earwig Forficula auricularia, (c-g) house cricket Acheta domesticus. (a, b) Frontal paraffin sections through the central body of $F$. auricularia at an intermediate (a) and more posterior (b) level. In addition to strong immunostaining in the lower division of the central body (CBL), the upper division shows immunostaining diffusely throughout the neuropil (GU1) and in a narrow layer (GU2). ML, medial lobe of the mushroom body. (c, d) Frontal reconstruction of GABA-immunoreactive neurons innervating the protocerebral bridge (PB, c) and the CBL (d) of A. domesticus. In (d), fan-like processes of major GU1 neurites in the upper division of the central body (CBU) are included. In the lateral complex, immunostaining is concentrated in two areas of microglomeruli, the medial bulb (MBU) and lateral bulb (LBU). Cell bodies are clustered antero-ventrally to the lateral accessory lobes (LAL). AL, antennal lobe. (e-g) Oblique sagittal section (e) and frontal sections at an anterior ( $f$ ) and posterior (g) level through the central body of $A$. domesticus. In addition to dense staining in the CBL, processes originating from an anterior commissure (arrowheads in e, $\mathrm{f}$ ) give rise to finely beaded terminals in an anterior dorsal layer of the CBU (GU1). Their major fibers divide this layer into 8 wedge-like domains, labeled 1-4 in g. Fibers originating from the posterior face of the CBL innervate a posterior layer (GU2) in a fan-like manner. a, anterior; p, posterior; NO, nodulus. Scale bars $=50 \mu \mathrm{m}$ in a (also applies to b); $100 \mu \mathrm{m}$ in d (also applies to c); $100 \mu \mathrm{m}$ in $\mathrm{f}$ (also applies to e, g)

the antennal lobes. Dense immunostaining originating from the isthmustract fibers was concentrated in small bilateral areas, the bulbs, immediately adjacent to the vertical lobes of the mushroom bodies. Microglomerular condensations in the bulbs were relatively inconspicuous. No staining was detected in the PB and noduli.

Of the three cockroach species studied, the patterns of immunostaining in the two blaberid species ( $R$. maderae, B. discoidalis) were virtually identical, and therefore, only staining in $R$. maderae is presented in Figure 5. Immunostaining in the CBL was extremely dense and apparently resulted from a particularly large number of immunostained neurons (Figure $5 c-f$ ). Staining in the CBL was patterned like a fan-shaped rosette consisting of eight cone-shaped domains (labeled 1-4 in Figure 5f) and nine smaller immunostained intercone domains laterally from the outermost cones and between adjacent cones (labeled a-e in Figure 5f). Staining of two fiber systems in the CBU (GU1, GU2) was very sparse and consisted of exceedingly fine processes. The bulbs were large, sharply delineated against the surrounding neuropil, and appeared to consist of numerous very small microglomerular condensations (Figure $5 c, d, f$ ). In all three cockroach species, the PB was tangentially penetrated by a few immunostained fine beaded processes (Figure $5 \mathrm{~g}, \mathrm{~h}$ ), whereas the noduli were unlabeled. In P. americana, immunostaining in the CBL was also dense, but immunostaining rather had a palisade-like appearance with a smooth boundary against the CBU (Figure 5i,j). Immunostaining in the
GU1 and GU2 systems was more obvious than in R. maderae, and the GU2 system had a fan-shaped appearance (labeled 1-3 in Figure 5j). Numerous immunostained cell bodies of the CB neurons were clustered in the anterior-medial protocerebrum near the antennal lobe (Figure 5k).

\section{5 | GABA immunostaining in the central complex of true bugs (Hemiptera)}

Four hemipteran species were studied, the water strider Notonecta glauca, the backswimmer Gerris lacustris, the shield bug Palomena prasina, and the assassin bug Psytalla horrida, all belonging to the suborder Heteroptera (Table 1, Figure 6). In all species the CBL showed dense GABA immunoreactivity, originating form immunostained tangential neurons with additional ramifications in the bulbs. As revealed by immunostaining, the shape of the CBL differed substantially between the four species. In N. glauca, the CBL had a semicircular appearance in frontal planes, with a less densely stained area in the center (Figure 6c). In G. lacustris, the CBL was even further transformed into a toroidal structure (Figure 6f,g). In contrast, in P. prasina and $P$. horrida, the $\mathrm{CBL}$ had a more conventional elongated, in $P$. horrida sausage-shaped appearance (Figure 6h,i). Ramifications in the bulbs were fine in N. glauca (Figure 6a,b) without evidence for microglomeruli, in the shield and assassin bug they consisted of 


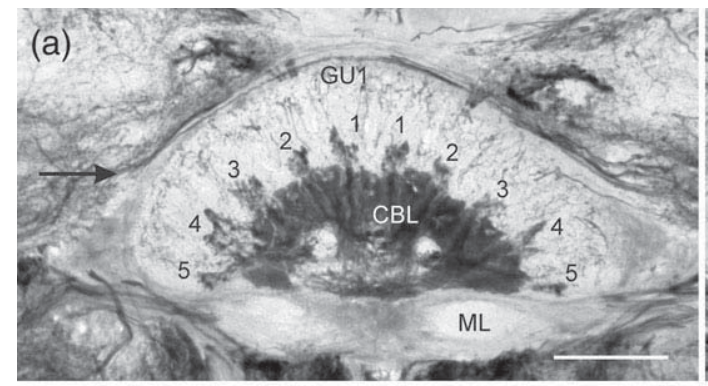

(c)

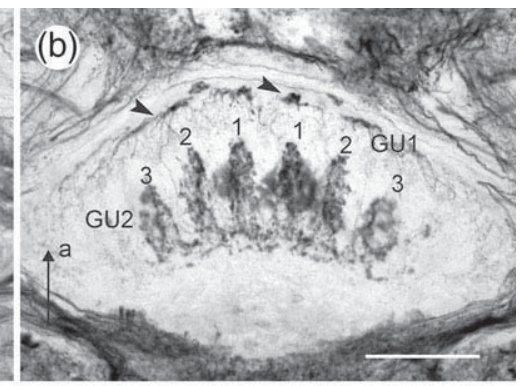

(d)
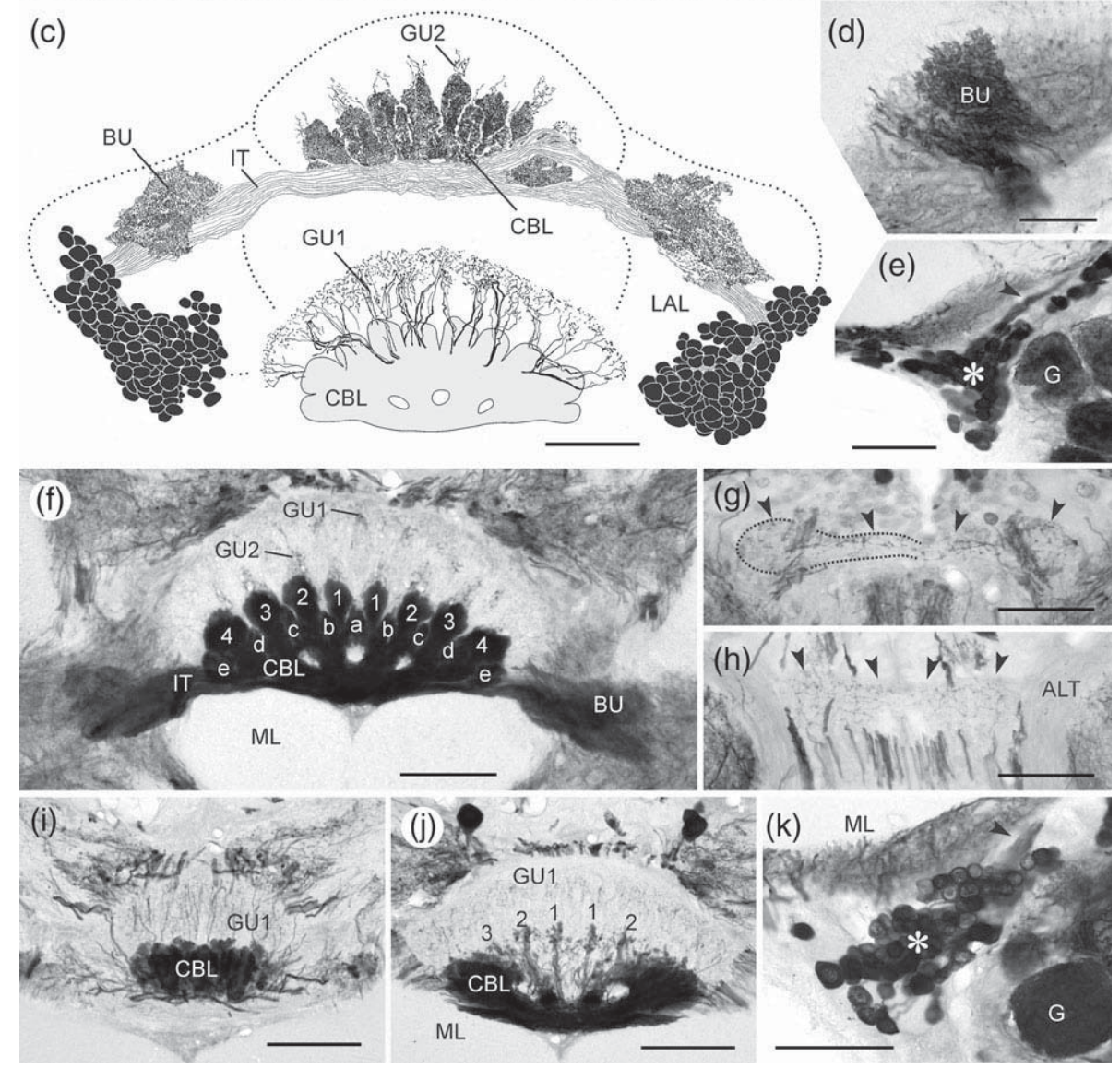

FIGURE 5 GABA immunostaining in the central complex of Dictyoptera. (a, b) Praying mantis Hierodula membranacea, (c-g) Madeira cockroach Rhyparobia maderae, (h-k) American cockroach Periplaneta americana. (a, b) Frontal (a) and horizontal (b) vibratome sections through the central body. The lower division of the central body (CBL) shows dense GABA immunostaining. Sparse staining in the upper division of the central body (CBU) is present in two fiber systems, diffusely branching neurons in the uppermost layer of the CBU (GU1) and, adjacent to the dorsal face of the CBL, staining arranged in 10 domains (GU2, labeled 1-5). Arrow in (a) points to level of section in (b). a, anterior; ML, medial lobe of the mushroom body. (c) Frontal reconstruction of GABA-immunoreactive neurons innervating the central body of the cockroach $R$. maderae. Cell bodies are clustered dorsomedial from the antennal lobe (AL). Neurons have dense ramifications in the bulbs (BU) and innervate the central body via massive isthmus tracts (IT). Staining in the CBL is extremely dense and is concentrated in cone-like domains. Sparse fibrous staining of GU2 neurons is concentrated in eight columns largely following the cone-like domains in the CBL. Inset shows reconstruction of the GU1 fiber system anterior to the CBL. (d) Detail of the right bulb (BU), illustrating numerous very small microglomerular condensations. (e) Cell body cluster (asterisk) near the AL. Arrowhead points to fascicle of cell body fibers. (f) Frontal vibratome section through the central body, IT and BU. In the $\mathrm{CBL}$ dense staining is concentrated in eight cone-like compartments (labeled 1-4) and nine domains between adjacent cones (labeled a-e). (g, h) Fine beaded processes (arrowheads) invade the PB in R. maderae (g) and P. americana (h). Dotted lines in (g) outline the left PB hemisphere. (i, j) Frontal sections through the central body of $P$. americana at an anterior (i) and more posterior (j) level. Dense immunostaining in the CBL is concentrated in knot-like structures. Anterior to the CBL, fibers project to terminals in a dorsal layer of the CBU (GU1). Posterior to the CBL processes of the GU2 system have a fan-shaped appearance (labeled 1-3). (k) Cell body cluster (asterisk) and fascicle of cell body fibers (arrowhead) dorsomedial to the AL. ALT, antennal lobe tract; G, antennal-lobe glomerulus; ML, medial lobe of the mushroom body. Scale bars $=100 \mu \mathrm{m}$ in a-c, e-k; $50 \mu \mathrm{m}$ in d

prominent, large microglomeruli (Figure 6h,i). Bulbs could not be identified in the backswimmer. Cell bodies connected via fibers in the isthmus tracts were clustered lateral to the lateral accessory lobes or between the lateral accessory lobes and antennal lobes.
In all species, two distinct layers were supplied by GABAimmunostained processes in the $\mathrm{CBU}$. The uppermost layer of the CBU was invaded diffusely by sparse beaded processes of GU1 neurons that originated from a small arcuate fascicle of fibers projecting 

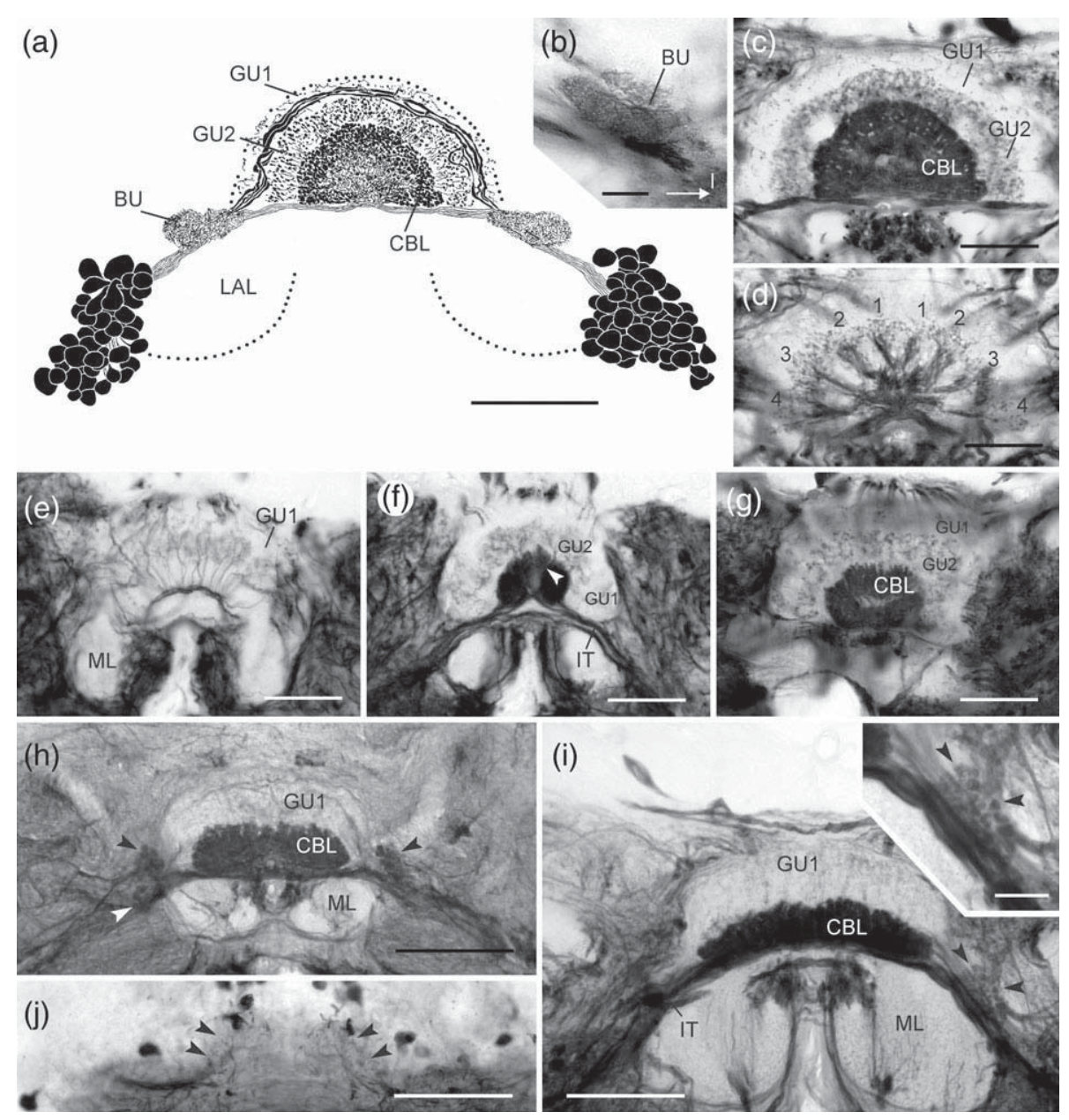

FIGURE 6 GABA immunostaining in the central complex of Hemiptera. (a-d) Water strider Notonecta glauca, (e-g) backswimmer Gerris lacustris, (h, j) green shield bug Palomena prasina, (i) assassin bug Psytalla horrida. (a) Frontal reconstruction of GABA-immunoreactive neurons innervating the central body. Immunostained neurons have cell bodies in bilateral clusters between the lateral accessory lobes and the antennal lobes. Immunostained fibers could be traced to two small areas ventrolateral from the central body, the bulbs (BU), which are densely innervated. Neurites enter the lower division of the central body $(\mathrm{CBL})$ ventrally and give rise to dense immunostaining throughout the $\mathrm{CBL}$. In addition, two layers of the upper division (CBU) show GABA immunoreactivity (GU1, GU2). The dorsalmost layer (GU1) is sparsely supplied by immunostained terminals derived from large fibers projecting along an arc in front of the CBU. (b) The bulb (BU) is densely innervated by fine GABAimmunoreactive processes. I, lateral. (c, d) Sections at intermediate (c) and posterior (d) levels through the central body. Staining of GU2 (d) originates from eight (1-4) fiber fascicles innervating the layer in a fan-like manner. (e, f) Adjacent frontal sections through the central body of a water strider. (e) An arched fiber fascicle gives rise to sparse immunostaining in the uppermost layer of the CBU (GU1). (f) The adjacent section shows dense immunostaining in the CBL and ascending fibers (arrowhead) of GU2 neurons in the CBU. (g) Horizontal section illustrating the toroidal shape of the CBL and immunostaining of GU2 neurons in the CBU. (h, i) In the green shield bug (h) and the assassin bug (i) the densely stained CBL has a more elongated shape than in Gerris and Notonecta. Particularly prominent are large microglomeruli in the bulbs (arrowheads) which in the shield bug are divided into two subfields. Inset in (i) shows enlargement of bulb with microglomeruli (arrowheads). (j) Sparse GABA immunostaining is present in the protocerebral bridge of $P$. prasina (arrowheads). IT, isthmus tract; ML, medial lobe of the mushroom body. Scale bars $=100 \mu \mathrm{m}$ in a, $\mathrm{h}-\mathrm{j} ; 25 \mu \mathrm{m}$ in b and in inset in i; $50 \mu \mathrm{m}$ in c-g

along the anterior face of the CBU (Figure 6a,h) or CBL (Figure 6e). Ramifications of these neurons outside the $C B$ could not be identified. A lower layer of the $\mathrm{CBU}$, adjacent to the upper margin of the $\mathrm{CBL}$, was more densely immunostained (Figure $6 a, c, d, f, g)$. In N. glauca, these arborizations, like the GABA-immunostained CBL neurons, originated from fibers in the isthmus tract and formed eight fascicles of fibers that ascended to their layer of innervation in a fan-like manner posterior to the CBL (Figure $6 \mathrm{~d}$ ). These neurons were labeled GU2. In G. lacustris, GU2 fibers projected through the GABA-negative canal in the toroidal CBL to their ramification sites in the CBU (Figure 6f,g). In the shield and assassin bug, this layer was small and largely posterior to the CBL. The PB could not be unequivocally identified in G. lacustris and N. glauca; in P. prasina and P. horrida, it was very sparsely supplied by fine beaded processes (Figure 6j).

\subsection{GABA immunostaining in the central complex of wasps, bees and ants (Hymenoptera)}

GABA-immunoreactivity was studied in the honeybee Apis mellifera, the wasps Vespula vulgaris and Paravespula germanica, and in the red wood ant Formica rufa (Figure 7). Immunostaining in worker (Figure 7a-c), queen (Figure 7d), and drone bee (not shown) showed 

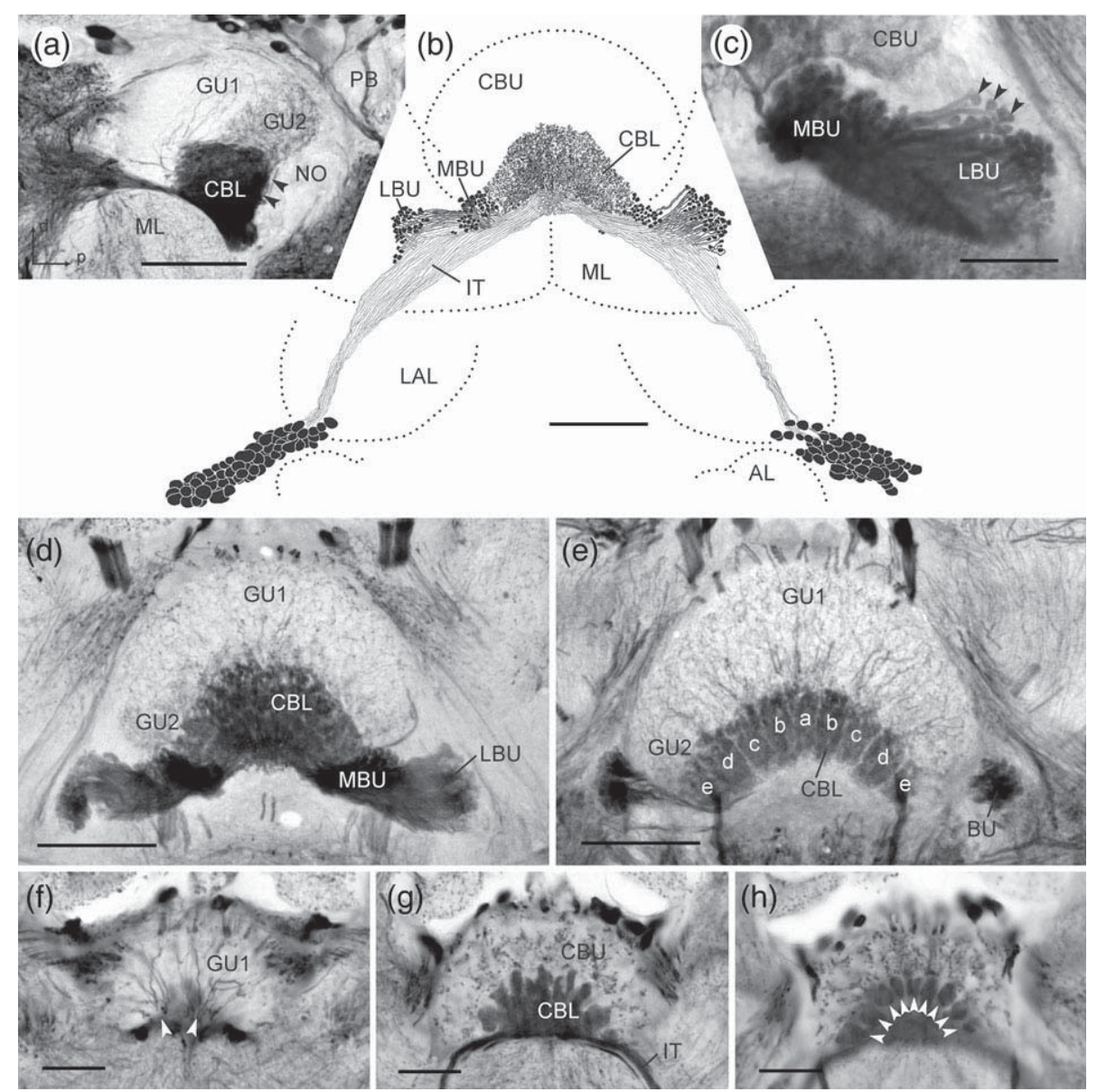

FIGURE 7 GABA immunostaining in the central complex of Hymenoptera. (a-d) Honeybee Apis mellifera, (e) German wasp Paravespula germanica, (f-h) red wood ant Formica rufa. (a) Sagittal section through the central complex of a worker bee. Dense immunostaining is present in the lower division of the central body (CBL), while the upper division is innervated sparsely by two systems of immunostained neurons (GU1, GU2). Neurites of GU1 originate from fibers in front of the CBL, while the GU2 fibers project from the fiber complex below the central body along the posterior face of the CBL (arrowheads). Noduli and the protocerebral bridge are free of immunostaining. (b) Frontal reconstruction of GABA immunoreactive neurons innervating the CBL of a worker bee. The neurons have cell bodies between the antennal lobe, the inferior protocerebrum, and the lateral accessory lobes (LAL). Their fibers innervate the lateral (LBU) and medial bulbs (MBU) and the CBL. (c) Details of immunostaining in the medial (MBU) and lateral (LBU) bulb. Microglomerular complexes (arrowheads) lie at the tips of stout GABA-

immunoreactive processes. (d) Frontal vibratome section showing GABA immunostaining in the central body, MBU and LBU of a queen bee. The upper division of the central body is, like in the worker bee, supplied by two types of tangential neurons (GU1, GU2). (e) Frontal section through the central body of a German wasp. Here, only a single bulb (BU) was recognized. The CBL shows, as in the bee, dense immunostaining, which, at this posterior level through the $C B L$ is arranged in nine modules (labeled a-e). As in the bee two systems of tangential neuron innervating the upper division of the central body (GU1, GU2), could be distinguished. (f-h) Consecutive frontal 30- $\mu \mathrm{m}$ vibratome sections through the central complex of a worker ant. In (f), fibers project anteriorly from the CBL (arrowheads) to the upper division of the central body, corresponding to GU1 neurons. (g) The CBL is densely supplied by GABA-immunoreactive processes originating from fibers in the isthmus tract (IT). (h) At a posterior level, nine modules of GABA staining (arrowheads) can be distinguished in the CBL. Scale bars $=100 \mu \mathrm{m}$ in a, b, d, e; $50 \mu \mathrm{m}$ in c, f-h

identical patterns. As in all other insect species, the CBL in all hymenopterans was densely invaded by GABA-immunostained tangential neurons with additional ramifications in the bulbs. Their cell bodies were arranged in an elongated cluster between the antennal lobe and the anterior ventrolateral protocerebrum (Figure 7b). In the honeybee, two separate areas of microglomerular condensations, the medial and lateral bulb, could be distinguished (Figure $7 b, c, d$ ) while in the wasps (Figure 7e) and the ant only a single bulb was present, corresponding in position to the lateral bulb of the bee. Cell body fibers in the isthmus tract of the bee occurred first as a single compact fascicle, but when reaching the level of the medial lobe of the mushroom body they continued as a flat sheet of fibers passing posteriorly around the massive medial lobes (Figure 7b). Processes in the bulbs were highly peculiar: relatively sturdy side branches from the fibers in the isthmus tract invaded the bulb and terminated with prominent single spheroidal microglomeruli (Figure 7c). Although less prominent, this arrangement was also present in the wasps and the ant. In the bee, some of these microglomeruli were isolated and in those cases seemed to be innervated only by a single process (Figure 7c). In F. rufa, GABA immunostaining in the $C B L$ had an irregular lobate appearance toward the CBU (Figure 7g), but near the posterior face of the CBL both in the wasp and the ant, a more regular arrangement of nine immunostained modules became apparent (Figure 7e,h).

In all hymenopterans studied, the CBU was innervated by two types of tangential neuron, GU1 and GU2, which entered the CBU from the strongly immunostained fiber system below the CBL. Fibers 
of GU1 neurons passed anteriorly around the CBL and invaded an intermediate layer of the $\mathrm{CBU}$, while GU2 neurons entered the $\mathrm{CBU}$ along the posterior face of the $\mathrm{CBL}$ and innervated a most posterior region of the $\mathrm{CBU}$ in a fan-like fashion (Figure 7a,e). In F. rufa, the innervation domains of the two fiber systems could not be clearly distinguished, because both gave rise to similarly beaded terminals in the CBU (Figure 7g,h). In all species studied, the noduli and PB were free of immunostaining (Figure 7a).

\subsection{GABA immunostaining in the central complex of beetles (Coleoptera)}

Immunostaining was analyzed in the sun beetle Pachnoda marginata and the june beetle Amphimallon solstitiale (Scarabeidae), the mealworm beetle Tenebrio molitor (Tenebrionidae), and the water beetle Acilius sulcatus (Dytiscidae) (Table 1, Figure 8). GABA immunostaining in all species was highly similar. The CBL had a hemiellipsoid shape and was densely supplied by GABA-immunoreactive processes from neurons with cell bodies between the antennal lobes and medial lobes of the mushroom body (Figure 8a,l,k). Side branches from their fibers in the isthmus tracts gave rise to particularly large microglomerular terminals in the bulbs (Figure 8a,f,h,i). The CBL showed irregular condensations of immunostaining but no regular pattern of layers or columns (Figure 8a,i,k).

As in other insects, two systems of tangential neuron of the CBU showed GABA immunostaining. GABA-labeled GU1 neurons innervated an anterior-dorsal layer of the $C B U$ with varicose terminals. In $P$. marginata and $A$. solstitiale their processes originated from two bilateral pairs of large neurons crossing the midline in front of the CBL (Figure $8 \mathrm{~b}, \mathrm{~d}, \mathrm{e}, \mathrm{g}$ ). In $\mathrm{P}$. marginata, their ramifications could be partly reconstructed (Figure $8 \mathrm{~d}$ ). Their axonal fibers originated from the isthmus tracts, but cell body location and dendritic processes could not be identified. In larvae of the water beetle $A$. sulcatus, GU1 neurons were the only GABA-immunostained neurons innervating the $C B$ (Figure 8j), indicating that the GABA-immunoreactive supply of the CBL as well as the GU2 neurons of the CBU develop during adult metamorphosis. Ramifications of GU2 neurons in the CBU were much finer than those of the GU1 neurons. Their processes originated from fibers ascending to the $C B U$ along the posterior face of the $C B L$ and gave rise to a patchy innervation of a CBU layer just posterior to the layer innervated by GU1 neurons (Figure 8c,g,i). In all species studied, the PB and noduli were free of immunostaining (Figure 8e).

\section{8 | GABA immunostaining in the central complex of Lepidoptera and a scorpionfly (Mecoptera)}

Immunostaining was studied in three lepidopteran species, the monarch butterfly Danaus plexippus, the owl butterfly Eryphanis polyxena and the sphinx moth Manduca sexta (Table 1, Figure 9). In all species, the CBL had an elongated bar-shaped appearance and was densely supplied by GABA-immunoreactive processes (Figure 9). Immunostaining in the CBL did not show a columnar organization but, especially in M. sexta, a distinct horizontal layering (Figure 9f). Staining in the $\mathrm{CBL}$ originated from bilateral clusters of neurons with cell bodies medially between the antennal lobes and lateral accessory lobes
(Figure 9a). Neurons gave rise to dense staining in microglomerular condensations in large bulbs, which in E. polyxena had almost the size of the medial lobes of the mushroom bodies. In E. polyxena and M. sexta, but less clearly in D. plexippus, the bulbs were subdivided into superior and inferior components (Figure 9d,g).

As in most other species studied, the CBU was invaded by two types of apparently tangential neurons, termed GU1 and GU2 (Figure 9b,e). In D. plexippus, two bilateral pairs of neurons from the isthmus tracts bypassed the CBL anterior-dorsally and gave rise to diffusely organized beaded terminals in a broad superior layer of the CBU. Their fiber paths and CBU innervation corresponds to two pairs of GABA-immunoreactive neurons in the CBU of $M$. sexta, described by Homberg et al. (1987). In addition, a narrow layer more ventrally in the CBU was innervated by GABA-immunostained GU2 neurons (for M. sexta see Homberg et al., 1987). Their fibers entered the CBU along the ventral surface of the CBL (Figure 9e). Noduli and protocerebal bridge in all species studied were free of immunolabeling.

Immunostaining in the scorpionfly, representing Mecoptera, was dense and had a patchy, palisade-like pattern in a hemiellipsoid to cresent-shaped CBL (Figure 9h). Somata of the CBL-innervating neurons were clustered mediodorsally from the antennal lobe. Neurons projected to prominent spheroidal microglomeruli in the bulb before entering the $\mathrm{CBL}$ (Figure $9 \mathrm{~h}$ ). The $\mathrm{CBU}$ was sparsely supplied by immunoreactive processes. Diffuse immunostaining throughout large parts of the CBU, probably corresponding to GU1 neurons, could be distinguished from a narrow immunoreactive layer, likely corresponding to GU2 neurons. PB and noduli were free of immunoreactivity.

\subsection{GABA immunostaining in the central complex of mosquitos and flies (Diptera)}

Immunostaining was studied in the yellow fever mosquito Aedes aegypti (Culicidae), the cranefly Tipula oleracea (Tipulidae), the blow fly Lucilia sericata (Calliphoridae), and the flesh fly Sarcophaga carnaria (Sarcophagidae) (Table 1, Figure 10). In all species, the CBL showed dense immunostaining (Figure 10). In the mosquito, the CBL had an almost triangular shape, in the cranefly it was nearly hemispheric, and in higher flies, the two lateral ends were bent down and approached each other resulting in a ventrally open toroid (Figure 10). In Drosophilidae, both ends are fused across the midline to a complete toroid resulting in the name "ellipsoid body" for the CBL (Ito et al., 2014). In all species studied here, GABA-immunoreactive fibers crossed the midline ventrally from the $\mathrm{CBL}$ to innervate the contralateral hemisphere of the CBL. In doing so, they gave rise to an unlabeled midline canal (Figure 10a,b,f,g), which is used as the entry site of anteriorbundle fibers into the CBU (Pfeiffer \& Homberg, 2014). As in all species studied so far, immunostaining in the CBL originated from neurons with cell bodies between the antennal lobe and lateral accessory lobe (Figure 10d,e). Before entering the CBL, the neurons gave rise to knot-like terminals in the bulbs, located in the notch between CBU and lateral accessory lobe (Figure 10d,f, inset in e). Axonal fibers entered the CBL anteriorly via the lateral ellipsoid tract (Figure 10d), corresponding to the isthmus tract in other insects. Immunostaining in 

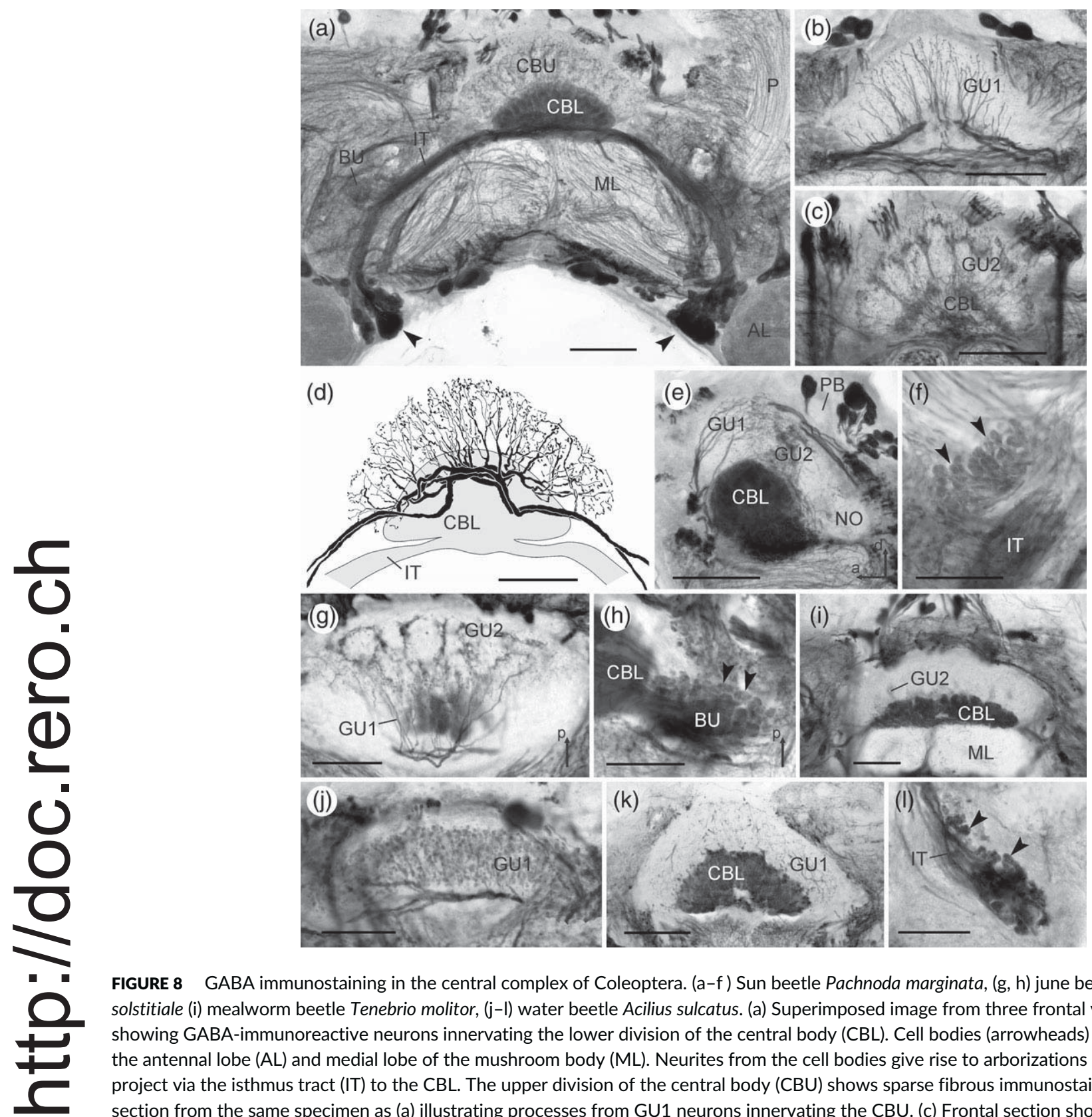

FIGURE 8 GABA immunostaining in the central complex of Coleoptera. (a-f) Sun beetle Pachnoda marginata, (g, h) june beetle Amphimallon solstitiale (i) mealworm beetle Tenebrio molitor, (j-I) water beetle Acilius sulcatus. (a) Superimposed image from three frontal vibratome sections showing GABA-immunoreactive neurons innervating the lower division of the central body (CBL). Cell bodies (arrowheads) are clustered between the antennal lobe (AL) and medial lobe of the mushroom body (ML). Neurites from the cell bodies give rise to arborizations in the bulbs (BU) and project via the isthmus tract (IT) to the CBL. The upper division of the central body (CBU) shows sparse fibrous immunostaining. (b) Frontal section from the same specimen as (a) illustrating processes from GU1 neurons innervating the CBU. (c) Frontal section showing fine processes of GU2 neurons ascending to the CBU posteriorly from the CBL. (d) Frontal reconstruction illustrating innervation of the CBU from two bilateral pairs of GU1 neurons anterior to the CBL. Their fibers join the IT toward their cell bodies. (e) Sagittal section illustrating immunostaining in the $\mathrm{CBL}$ and the GU1 and GU2 systems of the CBU. The protocerebral bridge (PB) and noduli (NO) are free of immunostaining. a, anterior; d, dorsal. (f) In the bulbs, the immunostained neurons of the CBL give rise to prominent microglomeruli (arrowheads). IT, isthmus tract. (g, h) Horizontal sections showing the GU1 and GU2 fiber systems in the june beetle at the dorsal margin of the CBL (g) and large microglomeruli (arrowheads) in the $\mathrm{BU}$ innervated by immunostained neurons of the CBL. p, posterior. (i) Frontal section through the central body of $T$. molitor showing dense immunostaining in the CBL and sparse innervation of the CBU by GU2 processes. ML, medial lobe of the mushroom body. (j) Superimposed image from two frontal sections through the central body of an A. sulcatus larva. A bilateral pair of GABA-immunostained GU1 neurons innervates the $\mathrm{CBU}$. The $\mathrm{CBL}$ and its associated GABA-immunoreactive neurons are absent. (k) Frontal section illustrating GABA immunostaining in the CBL and GU1 neurons of an imago. (I) Superimposed image from three adjacent vibratome sections. The large microglomeruli (arrowheads) of the BU show strong immunostaining. IT, isthmus tract. Scale bars $=100 \mu \mathrm{m}$ in a-e, $\mathrm{k} ; 50 \mu \mathrm{m}$ in $\mathrm{f}-\mathrm{j}, \mathrm{I}$

the $\mathrm{CBL}$ of the brachyceran species had a layered appearance (Figure 10d-f).

In all dipterans studied, the CBU was sparsely invaded by two types of tangential neuron, GU1 and GU2 (Figure 10b,c, inset in d, e, f-h). In the mosquito and cranefly, GU1 neurons gave rise to diffusely arborizing beaded projections in a broad most superior layer of the CBU (Figure 10b,c), while in Brachycera innervation of GU1 was exceedingly sparse and located in an intermediate layer of the CBU (Figure 10g,h). Staining of GU2 neurons was confined to a narrow layer ventrally in the CBU (Figure 10c, inset in d, e-h). While GU1 neurons entered the CBU along the anterior-dorsal face of the $\mathrm{CBU}$, fibers of GU2 neurons bypassed the CBL ventrally (Figure 10c) or took the CBL canal to invade the CBU (Figure 10g). The origin of both cell types could not be revealed. The PB and noduli were free of immunostaining. 


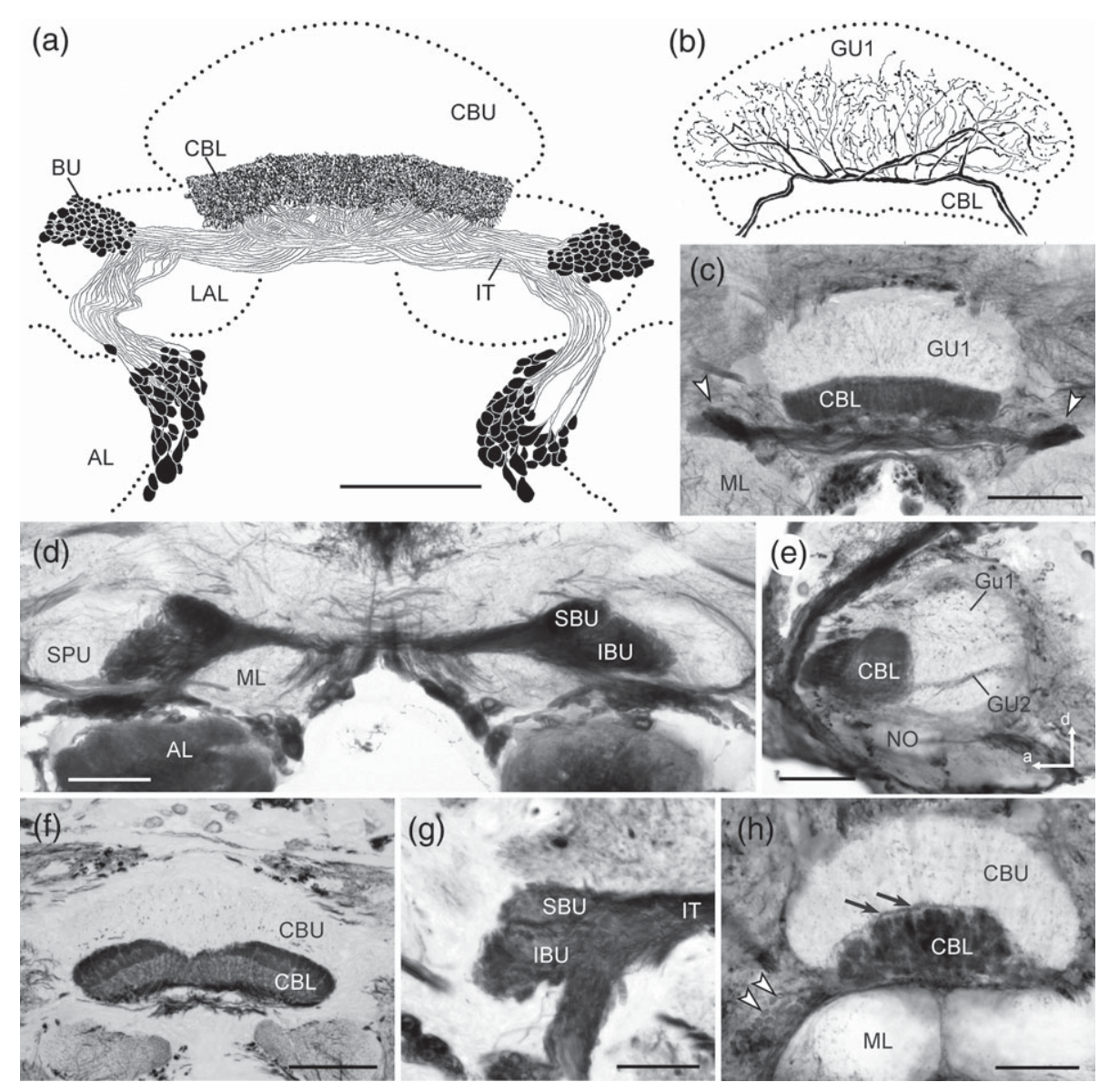

FIGURE 9 GABA immunostaining in the central complex of Lepidoptera and Mecoptera. (a-c) Monarch butterfly Danaus plexippus, (d, e) owl butterfly Eryphanis polyxena, (f, g) sphinx moth Manduca sexta, and (h) scorpionfly Panorpa communis. (a) Horizontal reconstruction (dorsal view) of GABA-immunoreactive neurons innervating the lower division of the central body (CBL). Cell bodies are clustered medially between the antennal lobe and lateral accessory lobe (LAL). (b) Reconstruction of arborizations of two bilateral pairs of GU1 neurons in the upper division of the central body (CBU). BU, bulb; IT, isthmus tract. (c) Horizontal vibratome section through the central body. Numerous immunostained neurons with dense ramifications in the bulbs (arrowheads) enter the CBL. The CBU shows sparse staining of GU1 neurons. ML, medial lobe of the mushroom body. (d) Horizontal vibratome section illustrating GABA immunoreactivity in large bulbs of the owl butterfly, subdivided into superior (SBU) and inferior (IBU) bulbs. SPU, spur of the mushroom body. (e) Sagittal section through the central body. Immunostained GU1 neurons give rise to fine beaded processes in the superior part of the CBU. Arborizations of immunolabeled GU2 neurons are confined to a narrow inferior layer in the CBU. The noduli (NO) are free of immunostaining. a, anterior; d, dorsal. (f) Horizontal paraffin section through the central body of the sphinx moth. Immunostaining in the CBL has a layered appearance. (g) Horizontal paraffin section illustrating the SBU and IBU in the sphinx moth. (h) Frontohorizontal vibratome section through the central body of the scorpionfly. Dense GABA immunostaining in the CBL has a patchy appearance. The $\mathrm{CBU}$ shows sparse immunostaining. Arrows point to emerging narrow layer of GU2 arborizations in the $\mathrm{CBU}$, arrowheads to microglomerular condensations in the bulb. Scale bars $=100 \mu \mathrm{m}$ in a-f; $50 \mu \mathrm{m}$ in $\mathrm{g}, \mathrm{h}$

\section{4 | DISCUSSION}

Across dicondylian insects, the pattern of GABA immunostaining in the central complex is highly conserved. In all species studied, the CBL showed dense labeling, which originated from neurons with cell bodies clustered between the antennal lobe and lateral complex. These neurons had ramifications in confined areas of the lateral complex, termed bulbs, and sent axons tangentially into the CBL. In addition, in all species studied in sufficient detail, two layers of immunolabeling could be distinguished in the CBU resulting from tangential neurons with cell bodies likely in the same cluster. These data point to a highly conserved neuronal organization of the $\mathrm{CB}$ and a common functional role of these neurons in the insect brain.

\subsection{Immunolabeled neurons of the CBL}

Based on the cell body positions of the immunostained neurons, their innervation of the bulb and CBL, it is likely that tangential neurons of the CBL (TL neurons) exhibit GABA immunoreactivity across dicondylian insects. In desert locusts, double labeling showed that two types of TL neurons, TL2 and TL3 are GABA-immunoreactive (Homberg et al., 1999). Similar neurons have been identified in the field cricket (Sakura et al., 2008), the monarch butterfly (Heinze et al., 2013; Heinze \& Reppert, 2011), a dung beetle (el Jundi et al., 2015), and two species of bees (Held et al., 2016; Stone et al., 2017). In the fruit fly, corresponding neurons were termed ring neurons (R-neurons), based on their ring-like innervation pattern in the ellipsoid body, and five subtypes R1-R5 were distinguished connecting the bulb with the ellipsoid body (Hanesch et al., 1989; Omoto et al., 2017). Judged from 

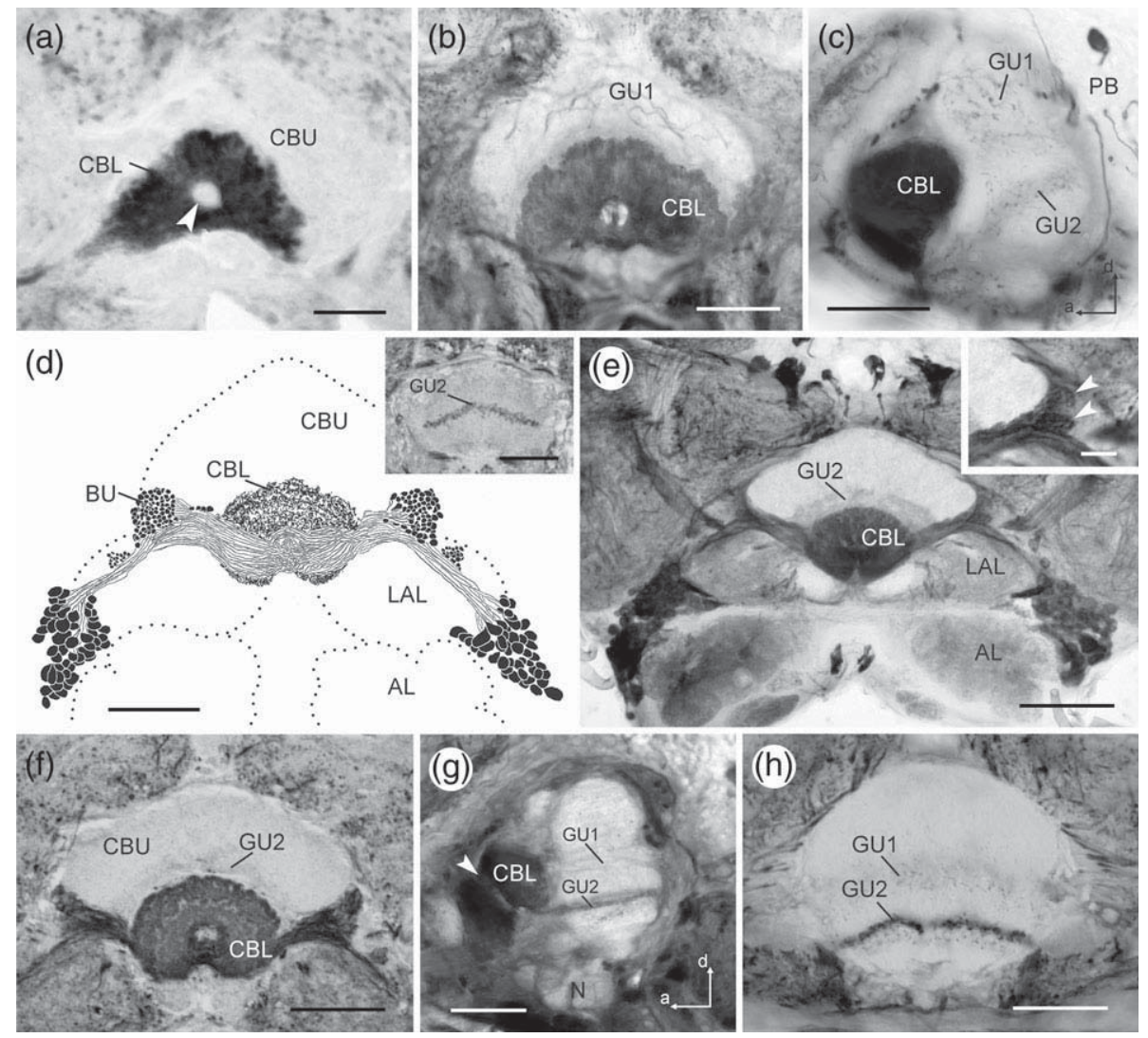

FIGURE 10 GABA immunostaining in the central complex of Diptera. (a) Yellow fever mosquito Aedes aegypti, (b, c) cranefly Tipula oleracea, (d, f) blow fly Lucilia sericata, (e, g, h) flesh fly Sarcophaga carnaria. (a) Superimposed image from two frontal paraffin sections showing GABA immunostaining in the lower division of the central body (CBL). Stained fibers pass below an immunonegative canal (arrowhead) to reach the contralateral hemisphere of the CBL. CBU, upper division of the central body. (b, c) Frontal (b) and sagittal (c) vibratome section through the central body of the cranefly showing dense GABA labeling in the CBL and sparse innervation of an upper (GU1) and a lower (GU2) layer of the $\mathrm{CBU}$. The protocerebral bridge (PB) is free of immunostaining. a, anterior; $d$, dorsal. (d) Frontal reconstruction of GABA-immunoreactive neurons innervating the $\mathrm{CBL}$ of the blow fly. Stained cell bodies are clustered between the antennal lobe (AL) and the lateral accessory lobe (LAL). Neurons densely innervate the bulbs (BU) with microglomerular complexes and enter the CBL fronto-ventrally. Inset shows GABA immunostaining in a narrow layer of the CBU corresponding to GU2 neurons. (e) Frontal vibratome section showing GABA immunostaining in the $\mathrm{CBL}$ and $\mathrm{CBU}$ of the flesh fly. Cell bodies are clustered between the $\mathrm{AL}$ and $\mathrm{LAL}$. In the $\mathrm{CBU}$, sparse fine staining above the CBL corresponds to GU2 neurons. Inset shows innervated bulb laterally from the CBU (arrowheads). (f) Frontal paraffin section illustrating GABA immunostaining in the central body of the blow fly. Staining in the CBL is arranged in concentric rings around an immunonegative canal. Arrowheads point to processes of immunolabeled GU2 neurons. $(\mathrm{g}, \mathrm{h})$. Sagittal $(\mathrm{g})$ and frontal $(\mathrm{h})$ sections through the central body of the flesh fly showing immunostaining in the CBU. GU1 neurons, entering the CBU from above the CBL innervate a median layer with exceedingly fine processes. GU2 neurons enter the $\mathrm{CBU}$ via the CBL canal (arrowhead) and form a narrow layer of beaded terminals in the inferior CBU. The noduli (N) are free of immunostaining. Scale bars $=20 \mu \mathrm{m}$ in a, inset in e; $50 \mu \mathrm{m}$ in b-d, inset in d, f-h; $100 \mu \mathrm{m}$ in e

GABA immunostaining the CBL in most species has an arcuate, crescent or bean-like shape, in large butterflies or dragonflies it is more elongated. More striking differences in shape occurred in Hemiptera and Diptera (Figures 6 and 10). Here the two lateral ends of the CBL were bent ventrally and are finally fused in the backswimmer (Figure 6g) and the fruit fly (Hanesch et al., 1989) forming a toroid. As a consequence, the arborizations of $\mathrm{R}$ neurons in Drosophila, in contrast to those of TL neurons of other insects, form a ring crossing the brain midline twice, in the dorsal and ventral part of the ellipsoid body (Omoto et al., 2017). The functional consequences of this modification for network connectivities in the central complex are still to be explored. Internally, GABA immunostaining revealed a layered organization of the $\mathrm{CBL}$ in many species such as the desert locust (Homberg et al., 1999), the honey bee (Figure 7b,d), the sphinx moth (Figure 9f), and flies (Figure 10d,f). Layering of the CBL is also well established in the desert locust and fruit fly at the level of single TL/R-neuron subtypes (Müller et al., 1997; Omoto et al., 2017). In other species a palisade-like organization was more prominent (Figures $3 b, 5 c, f$, and $7 \mathrm{e}, \mathrm{g}, \mathrm{h})$ reflecting the internal organization into 8/16 slices matching the postsynaptic columnar partners of TL/R neurons in the CBL. An interesting specialty was found in two nocturnal cockroaches, R. maderae and B. discoidalis. In both blaberids, GABA immunostaining was organized into eight cone-shaped domains and nine smaller intercone domains (Figure $5 f$ ). In the related $P$. americana (Blattidae), dense immunostaining in the $\mathrm{CBL}$ was rather uniformly distributed (Figure $5 \mathrm{i}, \mathrm{j}$ ). Because all three cockroach species showed GABA immunostaining in two systems of neurons of the CBU (GU1, GU2), we interpreted GABA immunostaining in the cone-like domains in 
R. maderae and B. discoidalis as part of the CBL. How this special organization of the $\mathrm{CBL}$ relates to connections with columnar neurons remains to be determined.

$T L / R$ neurons are the final stage of visual pathways conveying information on spatial orientation from the compound eye via the anterior optic tubercle to the central complex (Heinze, 2017; Pfeiffer \& Homberg, 2014; Turner-Evans \& Jayaraman, 2016). As shown in flies, locusts, bees, and ants, neurons from the anterior optic tubercle make synaptic contact with TL/R neurons in large microglomerular synaptic complexes in the bulbs each consisting of hundreds of active zones between presynaptic optic tubercle neurons and postsynaptic TL/R neurons (Held et al., 2016; Mota et al., 2016; Omoto et al., 2017; Schmitt et al., 2016; Träger et al., 2008). The significance of these peculiar connections is not known but they have been speculated to serve for fast and robust synaptic signaling and high spike time precision. The confinement of terminals of the immunoreactive CBL neurons to a small brain area, the bulb, underscores the universal presence of this pathway in dicondylian insects. Internally, however, the bulb differed in the organization of GABA immunolabeling. In many species the bulb was a uniform entity, in others it was subdivided into distinct areas (Figures 4d, 7b, 9d,g, and 10d) indicating distinct subtypes of TL/R neurons (Müller et al., 1997; Omoto et al., 2017). An internal organization into microglomeruli up to a size of small somata was found in most butterfly species. In contrast, in the firebrat, microglomeruli could not be identified, in the backswimmer, terminals in the bulb had a rather conventional, fine dendritic appearance, and in the cockroaches and mantis, microglomeruli were quite small. These observations indicate considerable differences in synaptic input organization of the immunolabeled CBL neurons.

\section{2 | Immunolabeled neurons of the CBU}

Sparse GABA immunostaining in selected layers of the $C B U$ has been reported in A. mellifera (Schäfer \& Bicker, 1986), M. sexta (Homberg et al., 1987), D. melanogaster (Hanesch et al., 1989), P. americana (Blechschmidt et al., 1990), A. domesticus (Strambi et al., 1998), S. gregaria (Homberg et al., 1999), and T. molitor (Wegerhoff, 1999). Extending those reports, we show here that in nearly all species studied, two systems of GABA-immunostained neurons can be distinguished in the CBU, termed GU1 and GU2, innervating different $\mathrm{CBU}$ layers. In all species that could be studied in detail, GU1 and GU2 innervations originate from axonal fibers in the isthmus tracts. Fibers of GU1 neurons pass the CBL anterodorsally and give rise to usually diffuse beaded terminals in a superior layer of the $C B U$, while processes of GU2 neurons pass around the CBL posterior-ventrally and extend side branches in a fan-like manner in an intermediate or inferior layer of the CBU. Partial reconstructions of GU1 neurons, whenever possible, revealed two bilateral pairs of neurons (Figures 8d,j and 9b; Homberg et al., 1987, 1999) illustrating a remarkable conservation across the studied species. GU2 neurons appeared to consist of a larger number of cells. While both GU1 and GU2 neurons have relatively large fiber diameters in locusts, beetles and butterflies/moths, their fiber diameters and terminals are considerably smaller in Hymenoptera and Diptera. Functional consequences of these differences will have to await physiological characterization of those neurons.

\section{3 | Developmental aspects}

The compact cluster of cell bodies of TL/R neurons, their coherent axonal trajectory in the isthmus tract and their common neurotransmitter strongly suggest that across dicondylian insects these neurons are clonally related. The origin of GU1 and GU2 arborizations from fibers in the isthmus tracts and their partial reconstructions, most detailed in the desert locust (Homberg et al., 1999) suggest that their cell bodies are located in the same cell cluster. In Drosophila, a particular lineage, EBa1, also termed DALv2, produces R-neurons connecting the bulb to the ellipsoid body (Wong et al., 2013; Yang et al., 2013). This lineage also contains a few neurons that sparsely ramify in two layers of the CBU/fan-shaped body, a broad upper layer and a narrow lower layer, corresponding in position to the two layers showing GABA immunostaining in larger flies (Figure 10). Interestingly, the upper fan-shaped body innervation, corresponding to GU1, originates from the first larval-born neurons of EBa1 (Yang et al., 2013).

While in Drosophila larva, the $\mathrm{CB}$ is completely absent, the CBU, but not the $\mathrm{CBL}$, is already present in the larva of other holometabolous species (Panov, 1959). The larval CBU in the tobacco hornworm M. sexta (Homberg \& Hildebrand, 1994), the meal worm T. molitor (Wegerhoff, 1999), and the diving beetle A. sulcatus (Figure 8j) show immunostaining in just two pairs of GU1 neurons, but still lack the apparently later developing GU2- and TL neurons. While meal worms and tobacco hornworms have no segmented legs, the diving beetle larva has six articulated legs just as the adult beetle and uses the typical hexapod tripod gait for locomotion under water (Nachtigall, 1962). These observations suggest that TL neurons and, in fact, the complete $\mathrm{CBL}$ are not essential for the control of walking and leg co-ordination.

\section{4 | Functional implications}

The insect central complex has been suggested to serve roles in decision making and action selection (Ritzmann et al., 2012; Sun et al., 2017; Wolff \& Strausfeld, 2016), control of locomotion (Martin, Guo, Mu, Harley, \& Ritzmann, 2015; Strauss, 2002), spatial orientation and navigation (Heinze, 2017; Pfeiffer \& Homberg, 2014; Turner-Evans \& Jayaraman, 2016; Varga, Kathman, Martin, Guo, \& Ritzmann, 2017), and, in Drosophila, promotion of sleep (Donlea et al., 2018; Liu et al., 2016). TL neurons provide sky compass information to the CBL in locusts, crickets, monarch butterflies, beetles, and bees (el Jundi et al., 2015; Heinze \& Reppert, 2011; Homberg, Heinze, Pfeiffer, Kinoshita \& el Jundi, 2011; Pegel, Pfeiffer, \& Homberg, 2018; Sakura et al., 2008; Stone et al., 2017), and R neurons, heading direction relative to a bright target in fruit flies (Seelig \& Jayaraman, 2013; Shiozaki \& Kazama, 2017). Detailed analysis in Drosophila is beginning to dissociate different functional roles of $\mathrm{R}$ neuron subtypes regarding their visual receptive fields, involvement in spatial memory, signaling selfmotion turns, and mediating sleep drive (Liu et al., 2016; Ofstad et al., 2011; Shiozaki \& Kazama, 2017).

Although the lifestyle of the studied species differed considerably and included migratory insects (locusts, monarch butterflies), central place foragers (bees, wasps, ants), flightless species (firebrat, worker ant), diurnal (bees, butterflies) and nocturnal species (cockroaches, sphinx moth), terrestrial (most species) and aquatic species (dragonfly 
larva, diving beetle), the input architecture to the CBL was highly conserved. This includes neuron morphology as well as the presumable neurotransmitter GABA and likely serves a universal role, perhaps in the selection of visual references for an internal space compass. Between different species, the elaboration of the system, however, differed as illustrated by the different size of microglomeruli in the bulbs, ranging from complete lack in water striders to particularly large size in beetles and butterflies, or the species-specific differences in the numbers of TL neurons. Plasticity of the system related to navigational needs is suggested by data from the highly visual ant Cataglyphis. Here the number of microglomeruli in the bulb significantly increased during transition from interior nest worker to forager leaving the nest (Schmitt et al., 2016). A functional role for TL neurons in compound-eye mediated visual operations may, finally, be concluded from a comparison of larval dragonflies and diving beetles. Dragonfly larvae have fully developed GABA immunostaining in the CBL just like all hemimetabolous insects (Figure 3), while the larva of the diving beetle $A$. sulcatus still lacks a CBL and associated GABA immunostaining (Figure 8). Both larvae predate on small aquatic animals, both have articulated legs used for swimming, but only dragonfly larvae have compound eyes, while A. sulcatus larvae have just six stemmata on each side (Schöne, 1951; Sherk, 1977).

\section{5 | Phylogeny}

Our comparative analysis suggests that GABA-immunoreactive neurons densely supplying the $\mathrm{CBL}$ and more sparsely two layers of the $\mathrm{CBU}$ are part of the evolutionary ground pattern of dicondylian insects. In contrast, sparse immunostaining of a few tangential neurons in the PB was only found in a cricket, cockroaches, and two hemipterans, but not in the earwig, mantid, and locust and might, thus, be of convergent evolutionary origin. It will be interesting to see, whether more basal hexapods like collembolans or diplurans show a staining pattern similar to Dicondylia. Both in collembolans and in diplurans, a CB is present (Böhm, Szucsich, \& Pass, 2012; Kollmann, Huetteroth, \& Schachtner, 2011). The dipluran species, however, seem to lack the division into a CBL and $\mathrm{CBU}$, and likewise in the collembolans studied, the $\mathrm{CB}$ rather resembles the CBU than the CBL. Both Diplura and Collembola are eyeless and may, thus, have lost the CBL secondarily. Notwithstanding the unresolved sister group of hexapods within Pancrustacea, insect-like central complexes exist in several crustacean taxa (Strausfeld, 1998; Utting, Agricola, Sandeman, \& Sandeman, 2000). While the CB in most groups is simple, resembling the insect CBU (Strausfeld, 1998), Thoen, Marshall, Wolff, and Strausfeld (2017) described a more elaborate CB in stomatopods consisting of an upper division and a lower division and accessory areas reminiscent of the insect bulbs. Unfortunately, the distribution of GABA has not been analyzed in any crustacean brain, but given its conserved distribution in the dicondylian central complex, research into this might provide valuable support for the systematic position of hexapods within Pancrustacea.

\section{ACKNOWLEDGMENTS}

We are grateful to Dr. Timothy Kingan for providing the anti-GABA antiserum and to Dr. Sabine Dietrich for providing butterfly species.
Funding was obtained from Deutsche Forschungsgemeinschaft, grant number: HO 950/24-1.

\section{ORCID}

Uwe Homberg (D) http://orcid.org/0000-0002-8229-7236

\section{REFERENCES}

Blechschmidt, K., Eckert, M., \& Penzlin, H. (1990). Distribution of GABA-like immunoreactivity in the central nervous system of the cockroach, Periplaneta americana (L.). Journal of Chemical Neuroanatomy, 3, 323-336.

Boer, H. H., Schot, L. P. C., Roubos, E. W., ter Maat, A., Lodder, J. C., \& Reichelt, D. (1979). ACTH-like immunoreactivity in two electrotonically coupled giant neurons in the pond snail Lymnaea stagnalis. Cell and Tissue Research, 202, 231-240.

Böhm, A., Szucsich, N. U., \& Pass, G. (2012). Brain anatomy in Diplura (Hexapoda). Frontiers in Zoology, 9, 26.

Buckingham, S. D., Biggin, P. C., Sattelle, B. M., Brown, L. A., \& Sattelle, D. B. (2005). Insect GABA receptors. Splicing, editing, and targeting by antiparasitics and insecticides. Molecular Pharmacology, 68, 942-951.

Donlea, J. M., Pimentel, D., Talbot, C. B., Kempf, A., Omoto, J. J., Hartenstein, V., \& Miesenböck, G. (2018). Recurrent circuitry for balancing sleep need and sleep. Neuron, 97, 378-389.

el Jundi, B., Warrant, E. J., Byrne, M. J., Khaldy, L., Baird, E., Smolka, J., \& Dacke, M. (2015). Neural coding underlying the cue preference for celestial orientation. Proceedings of the National Academy of Science of the United States of America, 112, 11395-11400.

Hanesch, U., Fischbach, K. F., \& Heisenberg, M. (1989). Neuronal architecture of the central complex in Drosophila melanogaster. Cell and Tissue Research, 257, 343-366.

Heinze, S. (2017). Unraveling the neural basis of insect navigation. Current Opinion in Insect Science, 24, 58-67.

Heinze, S., Florman, J., Asdokaraj, S., el Jundi, B., \& Reppert, S. M. (2013). Anatomical basis of sun compass navigation II: The neuronal composition of the central complex of the monarch butterfly. Journal of Comparative Neurology, 521, 267-298.

Heinze, S., \& Homberg, U. (2008). Neuroarchitecture of the central complex of the desert locust: Intrinsic and columnar neurons. Journal of Comparative Neurology, 511, 454-478.

Heinze, S., \& Reppert, S. M. (2011). Sun compass integration of skylight cues in migratory monarch butterflies. Neuron, 69, 345-358.

Held, M., Berz, A., Hensgen, R., Muenz, T. S., Scholl, C., Rössler, W., ... Pfeiffer, K. (2016). Microglomerular synaptic complexes in the sky-compass network of the honeybee connect parallel pathways from the anterior optic tubercle to the central complex. Frontiers in Behavioral Neurosciences, 10, 186.

Homberg, U. (2008). Evolution of the central complex in the arthropod brain with respect to the visual system. Arthropod Structure and Development, 37, 347-362.

Homberg, U., Heinze, S., Pfeiffer, K., Kinoshita, M., \& el Jundi, B. (2011). Central neural coding of sky polarization in insects. Philosophical Transactions of the Royal Society of London. Series B, Biological Sciences, 366, 680-687.

Homberg, U., \& Hildebrand, J. G. (1994). Postembryonic development of $\gamma$-aminobutyric acid-like immunoreactivity in the brain of the sphinx moth Manduca sexta. Journal of Comparative Neurology, 339, 132-149.

Homberg, U., Kingan, T. G., \& Hildebrand, J. G. (1987). Immunocytochemistry of GABA in the brain and suboesophageal ganglion of Manduca sexta. Cell and Tissue Research, 248, 1-24.

Homberg, U., Vitzthum, H., Müller, M., \& Binkle, U. (1999). Immunocytochemistry of GABA in the central complex of the locust Schistocerca gregaria: Identification of immunoreactive neurons and colocalization with neuropeptides. Journal of Comparative Neurology, 409, 495-507.

Hoskins, S. G., Homberg, U., Kingan, T. G., \& Hildebrand, J. G. (1986). Immunocytochemistry of GABA in the antennal lobes of the sphinx moth Manduca sexta. Cell and Tissue Research, 244, 243-252. 
Ito, K., Shinomiya, K., Ito, M., Armstrong, J. D., Boyan, G., Hartenstein, V. ... Vosshall, L. B. (2014). A systematic nomenclature for the insect brain. Neuron, 81, 755-765.

Kollmann, M., Huetteroth, W., \& Schachtner, J. (2011). Brain organization in Collembola (springtails). Arthropod Structure and Development, 40, 304-316.

Kunst, M., Pförtner, R., Aschenbrenner, K., \& Heinrich, R. (2011). Neurochemical architecture of the central complex related to its function in the control of grasshopper acoustic communication. PLoS One, 6, e25613.

Liu, G., Seiler, H., Wen, A., Zars, T., Ito, K., Wolf, R., ... Liu, L. (2006). Distinct memory traces for two visual features in the Drosophila brain Nature, 439, 551-556.

Liu, S., Liu, Q., Tabucchi, M., \& Wu, M. N. (2016). Sleep drive is encoded by neural plastic changes in a dedicated circuit. Cell, 165 1347-1360.

Lummis, S. C. R. (1990). GABA-receptors in insects. Comparative Biochemistry and Physiology, 95C, 1-8.

Martin, J. P., Guo, P., Mu, L., Harley, C. M., \& Ritzmann, R. E. (2015). Central-complex control of movement in the freely walking cockroach. Current Biology, 25, 2795-2803.

Meyer, E. P., Matute, C., Streit, P., \& Nässel, D. R. (1986). Insect optic lobe neurons identifiable with monoclonal antibodies to GABA. Histochemistry, 84, 207-216.

Misof, B., Liu, S., Meusemann, K., Peters, R. S., Donath, A., Mayer, C., ... Zhou, X. (2014). Phylogenomics resolves the timing and pattern of insect evolution. Science, 346, 763-767.

Mota, T., Kreissl, S., Durán, A. C., Lefer, D., Galizia, G., \& Giurfa, M. (2016). Synaptic organization of microglomerular clusters in the lateral and medial bulbs of the honeybee brain. Frontiers in Neuroanatomy, 10, 103.

Müller, M., Homberg, U., \& Kühn, A. (1997). Neuroarchitecture of the lower division of the central body in the brain of the locust Schistocerca gregaria. Cell and Tissue Research, 288, 159-176.

Nachtigall, W. (1962). Die Mechanik der Schwimmbewegungen bei der Acilius-Larve (Coleoptera, Dytiscidae). Internationale Revue der Gesamten Hydrobiologie, 47, 413-430.

Neuser, K., Triphan, T., Mronz, M., Poeck, B., \& Strauss, R. (2008). Analysis of a spatial orientation memory in Drosophila. Nature, 453 , 1244-1247.

Ofstad, T. A., Zuker, C. S., \& Reiser, M. B. (2011). Visual place learning in Drosophila melanogaster. Nature, 447, 204-207.

Omoto, J. J., Keles, M. F., Nguyen, B.-C. M., Bolanos, C., Lovick, J. K., Frye, M. A., \& Hartenstein, V. (2017). Visual input to the Drosophila central complex by developmentally and functionally distinct neuronal populations. Current Biology, 27, 1098-1110.

Panov, A. A. (1959). Structure of the insect brain at successive stages of postembryonic development. II. The central body. Entomological Review, 38, 276-283.

Pegel, U., Pfeiffer, K., \& Homberg, U. (2018). Integration of celestial compass cues in the central complex of the locust brain. Journal of Experimental Biology, 221, jeb171207.

Pfeiffer, K., \& Homberg, U. (2014). Organization and functional roles of the central complex in the insect brain. Annual Review of Entomology, 59, 165-184.

Poeck, B., Triphan, T., Neuser, K., \& Strauss, R. (2008). Locomotor control by the central complex in Drosophila-An analysis of the tay bridge mutant. Developmental Neurobiology, 68, 1046-1058.

Ritzmann, R. E., Harley, C. M., Daltorio, K. A., Tietz, B. R., Pollack, A. J. Bender, J. A., ... Quinn, R. D. (2012). Deciding which way to go: How do insects alter movements to negotiate barriers? Frontiers in Neuroscience, 6, 97.

Rosner, R., von Hadeln, J., Salden, T., \& Homberg, U. (2017). Anatomy of the lobula complex in the brain of the praying mantis compared to the lobula complexes of the locust and cockroach. Journal of Comparative Neurology, 525, 2343-2357

Sakura, M., Lambrinos, D., \& Labhart, T. (2008). Polarized skylight navigation in insects: Model and electrophysiology of e-vector coding by neurons in the central complex. Journal of Neurophysiology, 99, 667-682.
Schäfer, S., \& Bicker, G. (1986). Distribution of GABA-like immunoreactivity in the brain of the honeybee. Journal of Comparative Neurology, 246 , 287-300.

Schmitt, F., Stieb, S. M., Wehner, R., \& Rössler, W. (2016). Experience-related reorganization of giant synapses in the lateral complex: Potential role in plasticity of the sky-compass pathway in the desert ant Cataglyphis fortis. Developmental Neurobiology, 76, 390-404.

Schöne, H. (1951). Die Lichtorientierung der Larven von Acilius sulcatus L. and Dytiscus marginalis L. Zeitschrift für Vergleichende Physiologie, 33, 63-98.

Seelig, J. D., \& Jayaraman, V. (2013). Feature detection and orientation tuning in the Drosophila central complex. Nature, 503 262-266.

Seelig, J. D., \& Jayaraman, V. (2015). Neural dynamics for landmark orientation and angular path integration. Nature, 521, 186-191.

Sherk, T. E. (1977). Development of the compound eyes of dragonflies (Odonata). I. Larval compound eyes. Journal of Experimental Zoology, 201, 391-416.

Shiozaki, H. M., \& Kazama, H. (2017). Parallel encoding of recent visual experience and self-motion during navigation in Drosophila. Nature Neuroscience, 20, 1395-1403.

Sternberger, L. A. (1979). Immunocytochemistry. New York, NY: John Wiley and Sons.

Stone, T., Webb, B., Adden, A., Weddig, N. B., Honkanen, A., Templin, R., ... Heinze, S. (2017). An anatomically constrained model for path integration in the bee brain. Current Biology, 27, 1-17.

Strambi, C., Cayre, M., Sattelle, D. B., Augier, R., Charpin, P., \& Strambi, A. (1998). Immunocytochemical mapping of an RDL-like GABA receptor subunit and of GABA in brain structures related to learning and memory in the cricket Acheta domesticus. Learning and Memory, 5, 78-89.

Strausfeld, N. J. (1976). Atlas of an insect brain. Heidelberg, Germany: Springer.

Strausfeld, N. J. (1998). Crustacean - insect relationships: The use of brain characters to derive phylogeny amongst segmented invertebrates. Brain, Behavior and Evolution, 52, 186-206.

Strauss, R. (2002). The central complex and the genetic dissection of behavior. Current Opinion in Neurobiology, 12, 633-638.

Sun, Y., Nern, A., Franconville, R., Dana, H., Schreiter, E. R., Looger, L. L., ... Jayaraman, V. (2017). Neural signatures of dynamic stimulus selection in Drosophila. Nature Neuroscience, 20, 1104-1113.

Thoen, H. H., Marshall, J., Wolff, G. H., \& Strausfeld, N. J. (2017). Insect-like organization of the stomatopod central complex: Functional and phylogenetic implications. Frontiers in Behavioral Neuroscience, $11,12$.

Träger, U., Wagner, R., Bausenwein, B., \& Homberg, U. (2008). A novel type of microglomerular synaptic complex in the polarization vision pathway of the locust brain. Journal of Comparative Neurology, 205, 288-300.

Trautwein, M. D., Wiegmann, B. M., Beutel, R., Kjaer, K. M., \& Yeates, D. K. (2012). Advances in insect phylogeny at the dawn of the postgenomic area. Annual Review of Entomology, 57, 449-468.

Triphan, T., Poeck, B., Neuser, K., \& Strauss, R. (2010). Visual targeting of motor actions in climbing Drosophila. Current Biology, 20, 663-668.

Turner-Evans, D. B., \& Jayaraman, V. (2016). The insect central complex. Current Biology, 26, R445-R460.

Utting, M., Agricola, H. J., Sandeman, R., \& Sandeman, D. (2000). Central complex in the brain of crayfish and its possible homology with that of insects. Journal of Comparative Neurology, 416, 245-261.

Varga, A. G., Kathman, N. D., Martin, J. P., Guo, P., \& Ritzmann, R. E. (2017). Spatial navigation and the central complex: Sensory acquisition, orientation, and motor control. Frontiers in Behavioral Neuroscience, $11,4$.

Varga, A. G., \& Ritzmann, R. E. (2016). Cellular basis of head direction and contextual cues in the insect brain. Current Biology, 26, 1816-1828.

Vitzthum, H., Müller, M., \& Homberg, U. (2002). Neurons of the central complex of the locust Schistocerca gregaria are sensitive to polarized light. Journal of Neuroscience, 22, 1114-1125. 
Wang, Z., Pan, Y., Li, W., Jiang, H., Chatzmanolis, L., Chang, J., ... Liu, L. (2008). Visual pattern memory requires foraging function in the central complex of Drosophila. Learning and Memory, 15, 133-142.

Wegerhoff, R. (1999). GABA and serotonin immunoreactivity during postembryonic brain development in the beetle Tenebrio molitor. Microscopy Research and Technique, 45, 154-164.

Wolff, G., \& Strausfeld, N. J. (2016). The insect brain: A commentated primer. In A. Schmidt-Rhaesa, S. Harzsch, \& G. Purschke (Eds.), Structure and evolution of invertebrate nervous systems (pp. 597-639). Oxford, England: Oxford University Press.

Wolff, T., lyer, N. A., \& Rubin, G. M. (2015). Neuroarchitecture and neuroanatomy of the Drosophila central complex: A GAL4-based dissection of protocerebral bridge neurons and circuits. Journal of Comparative Neurology, 523, 997-1037.

Wong, D. C., Lovick, J. K., Ngo, K. T., Borisuthirattana, W., Omoto, J. J., \& Hartenstein, V. (2013). Postembryonic lineages of the Drosophila brain:
II. Identification of lineage projection patterns based on MARCM clones. Developmental Biology, 384, 258-289.

Yang, J. S., Awasaki, T., Yu, H. H., He, Y., Ding, P., Kao, J. C., \& Lee, T. (2013). Diverse neuronal lineages make stereotyped contributions to the Drosophila locomotor control center, the central complex. Journal of Comparative Neurololgy, 521, 2645-2662.

Young, J. M., \& Armstrong, J. D. (2010). Structure of the adult central complex in Drosophila: Organization of distinct neuronal subsets. Journal of Comparative Neurology, 518, 1500-1524. 